\title{
Strong Convergence for Generalized Equilibrium Problems, Fixed Point Problems and Relaxed Cocoercive Variational Inequalities
}

\author{
Chaichana Jaiboon ${ }^{1,2}$ and Poom Kumam ${ }^{1}$ \\ ${ }^{1}$ Department of Mathematics, Faculty of Science, King Mongkut's University of Technology Thonburi, \\ KMUTT, Bangkok 10140, Thailand \\ ${ }^{2}$ Department of Mathematics, Faculty of Applied Liberal Arts, Rajamangala University of Technology, \\ Rattanakosin, RMUTR, Bangkok 10100, Thailand
}

Correspondence should be addressed to Poom Kumam, poom.kum@kmutt.ac.th

Received 31 October 2009; Accepted 1 February 2010

Academic Editor: Jong Kim

Copyright (C) 2010 C. Jaiboon and P. Kumam. This is an open access article distributed under the Creative Commons Attribution License, which permits unrestricted use, distribution, and reproduction in any medium, provided the original work is properly cited.

We introduce a new iterative scheme for finding the common element of the set of solutions of the generalized equilibrium problems, the set of fixed points of an infinite family of nonexpansive mappings, and the set of solutions of the variational inequality problems for a relaxed $(u, v)$ cocoercive and $\xi$-Lipschitz continuous mapping in a real Hilbert space. Then, we prove the strong convergence of a common element of the above three sets under some suitable conditions. Our result can be considered as an improvement and refinement of the previously known results.

\section{Introduction}

Variational inequalities introduced by Stampacchia [1] in the early sixties have had a great impact and influence in the development of almost all branches of pure and applied sciences. It is well known that the variational inequalities are equivalent to the fixed point problems. This alternative equivalent formulation has been used to suggest and analyze in variational inequalities. In particular, the solution of the variational inequalities can be computed using the iterative projection methods. It is well known that the convergence of a projection method requires the operator to be strongly monotone and Lipschitz continuous. Gabay [2] has shown that the convergence of a projection method can be proved for cocoercive operators. Note that cocoercivity is a weaker condition than strong monotonicity.

Equilibrium problem theory provides a novel and unified treatment of a wide class of problems which arise in economics, finance, image reconstruction, ecology, transportation, network, elasticity, and optimization which has been extended and generalized in many directions using novel and innovative technique; see [3, 4]. Related to the equilibrium 
problems, we also have the problem of finding the fixed points of the nonexpansive mappings. It is natural to construct a unified approach for these problems. In this direction, several authors have introduced some iterative schemes for finding a common element of a set of the solutions of the equilibrium problems and a set of the fixed points of infinitely (finitely) many nonexpansive mappings; see [5-7] and the references therein. In this paper, we suggest and analyze a new iterative method for finding a common element of a set of the solutions of generalized equilibrium problems and a set of fixed points of an infinite family of nonexpansive mappings and the set solution of the variational inequality problems for a relaxed $(u, v)$-cocoercive mapping in a real Hilbert space.

Let $H$ be a real Hilbert space and let $E$ be a nonempty closed convex subset of $H$ and $P_{E}$ is the metric projection of $H$ onto $E$. Recall that a mapping $f: E \rightarrow E$ is contraction on $E$ if there exists a constant $\alpha \in(0,1)$ such that $\|f(x)-f(y)\| \leq \alpha\|x-y\|$ for all $x, y \in E$. A mapping $S$ of $E$ into itself is called nonexpansive if $\|S x-S y\| \leq\|x-y\|$ for all $x, y \in E$. We denote by $F(S)$ the set of fixed points of $S$, that is, $F(S)=\{x \in E: S x=x\}$. If $E \subset H$ is nonempty, bounded, closed, and convex and $S$ is a nonexpansive mapping of $E$ into itself, then $F(S)$ is nonempty; see, for example, [8]. We recalled some definitions as follows.

Definition 1.1. Let $B: E \rightarrow H$ be a mapping. Then one has the following.

(1) $B$ is called monotone if $\langle B x-B y, x-y\rangle \geq 0$, for all $x, y \in E$.

(2) $B$ is called $v$-strongly monotone if there exists a positive real number $v$ such that

$$
\langle B x-B y, x-y\rangle \geq v\|x-y\|^{2}, \quad \forall x, y \in E
$$

(3) $B$ is called $\xi$-Lipschitz continuous if there exists a positive real number $\xi$ such that

$$
\|B x-B y\| \leq \xi\|x-y\|, \quad \forall x, y \in E .
$$

(4) $B$ is called $\eta$-inverse-strongly monotone, $[9,10]$ if there exists a positive real number $\eta$ such that

$$
\langle B x-B y, x-y\rangle \geq \eta\|B x-B y\|^{2}, \quad \forall x, y \in E .
$$

If $\eta=1$, we say that $B$ is firmly nonexpansive. It is obvious that any $\eta$-inversestrongly monotone mapping $B$ is monotone and $(1 / \eta)$-Lipschitz continuous.

(5) $B$ is called relaxed $(u, v)$-cocoercive if there exists a positive real number $u, v$ such that

$$
\langle B x-B y, x-y\rangle \geq(-u)\|B x-B y\|^{2}+v\|x-y\|^{2}, \quad \forall x, y \in E .
$$

For $u=0, B$ is $v$-strongly monotone. This class of maps is more general than the class of strongly monotone maps. It is easy to see that we have the following implication: $v$-strongly monotonicity $\Rightarrow$ relaxed $(u, v)$-cocoercivity. 
(6) A set-valued mapping $T: H \rightarrow 2^{H}$ is called monotone if for all $x, y \in H, f \in T x$ and $g \in T y$ imply $\langle x-y, f-g\rangle \geq 0$. A monotone mapping $T: H \rightarrow 2^{H}$ is maximal if the graph of $G(T)$ of $T$ is not properly contained in the graph of any other monotone mapping. It is known that a monotone mapping $T$ is maximal if and only if for $(x, f) \in H \times H,\langle x-y, f-g\rangle \geq 0$ for every $(y, g) \in G(T)$ implies $f \in T x$.

Let $B$ be a monotone mapping of $E$ into $H$ and let $N_{E} w_{1}$ be the normal cone to $E$ at $w_{1} \in E$, that is,

$$
N_{E} w_{1}=\left\{w \in H:\left\langle\vartheta-w_{1}, w\right\rangle \geq 0, \forall \vartheta \in E\right\}
$$

Define

$$
T w_{1}= \begin{cases}B w_{1}+N_{E} w_{1}, & \text { if } w_{1} \in E \\ \emptyset, & \text { if } w_{1} \notin E .\end{cases}
$$

Then $T$ is the maximal monotone and $0 \in T w_{1}$ if and only if $w_{1} \in \operatorname{VI}(E, B)$; see $[11,12]$

In addition, let $D: E \rightarrow H$ be a inverse-strongly monotone mapping. Let $F$ be a bifunction of $E \times E$ into $\mathbb{R}$, where $\mathbb{R}$ is the set of real numbers. The generalized equilibrium problem for $F: E \times E \rightarrow \mathbb{R}$ is to find $x \in E$ such that

$$
F(x, y)+\langle D x, y-x\rangle \geq 0, \quad \forall y \in E .
$$

The set of such $x \in E$ is denoted by $\operatorname{EP}(F, D)$, that is,

$$
\mathrm{EP}(F, D)=\{x \in E: F(x, y)+\langle D x, y-x\rangle \geq 0, \forall y \in E\}
$$

\section{Special Cases}

(I) If $D \equiv 0$ (:the zero mapping), then the problem (1.7) is reduced to the equilibrium problem:

$$
\text { Find } x \in E \text { such that } F(x, y) \geq 0, \quad \forall y \in E \text {. }
$$

The set of solutions of (1.9) is denoted by $\mathrm{EP}(F)$, that is,

$$
\mathrm{EP}(F)=\{x \in E: F(x, y) \geq 0, \forall y \in E\}
$$

(II) If $F \equiv 0$, the problem (1.7) is reduced to the variational inequality problem:

$$
\text { Find } x \in E \text { such that }\langle D x, y-x\rangle \geq 0, \quad \forall y \in E \text {. }
$$

The set of solutions of (1.11) is denoted by $\operatorname{VI}(E, D)$, that is,

$$
\mathrm{VI}(E, D)=\{x \in E:\langle D x, y-x\rangle \geq 0, \forall y \in E\} .
$$


The generalized equilibrium problem (1.7) is very general in the sense that it includes, as special case, some optimization, variational inequalities, minimax problems, the Nash equilibrium problem in noncooperative games, economics, and others (see, e.g., $[4,13]$ ). Some methods have been proposed to solve the equilibrium problem and the generalized equilibrium problem; see, for instance, [5, 14-28]. Recently, Combettes and Hirstoaga [29] introduced an iterative scheme of finding the best approximation to the initial data when $\mathrm{EP}(F)$ is nonempty and proved a strong convergence theorem. Very recently, Moudafi [24] introduced an itertive method for finding an element of $\operatorname{EP}(F, D) \cap F(S)$, where $D: E \rightarrow H$ is an inverse-strongly monotone mapping and then proved a weak convergence theorem.

For finding a common element of the set of fixed points of a nonexpansive mapping and the set of solutions of variational inequality problem for an $\eta$-inverse-strongly monotone, Takahashi and Toyoda [30] introduced the following iterative scheme:

$$
\begin{gathered}
x_{0} \in E \text { chosen arbitrary, } \\
x_{n+1}=\alpha_{n} x_{n}+\left(1-\alpha_{n}\right) \operatorname{SP}_{E}\left(x_{n}-\tau_{n} B x_{n}\right), \quad \forall n \geq 0,
\end{gathered}
$$

where $B$ is an $\eta$-inverse-strongly monotone mapping, $\left\{\alpha_{n}\right\}$ is a sequence in $(0,1)$, and $\left\{\tau_{n}\right\}$ is a sequence in $(0,2 \eta)$. They showed that if $F(S) \cap \operatorname{VI}(E, B)$ is nonempty, then the sequence $\left\{x_{n}\right\}$ generated by (1.13) converges weakly to some $z \in F(S) \cap \operatorname{VI}(E, B)$. On the other hand, Shang et al. [31] introduced a new iterative process for finding a common element of the set of fixed points of a nonexpansive mapping and the set of solutions of the variational inequality problem for a relaxed $(u, v)$-cocoercive mapping in a real Hilbert space. Let $S: E \rightarrow E$ be a nonexpansive mapping. Starting with arbitrary initial $x_{1} \in E$, defined sequences $\left\{x_{n}\right\}$ recursively by

$$
x_{n+1}=\alpha_{n} f\left(x_{n}\right)+\beta_{n} x_{n}+\gamma_{n} \mathrm{SP}_{E}\left(I-\tau_{n} B\right) x_{n}, \quad \forall n \geq 1 .
$$

They proved that under certain appropriate conditions imposed on $\left\{\alpha_{n}\right\},\left\{\beta_{n}\right\},\left\{\gamma_{n}\right\}$, and $\left\{\tau_{n}\right\}$,

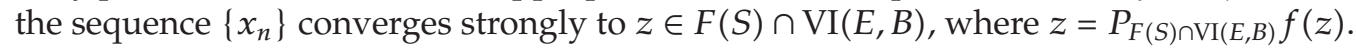

In 2008, S. Takahashi and W. Takahashi [27] introduced the following iterative scheme for finding an element of $F(S) \cap \operatorname{EF}(F, D)$ under some mild conditions. Let $E$ be a nonempty closed convex subset of a real Hilbert space $H$. Let $D$ be an $\eta$-inverse-strongly monotone mapping of $E$ into $H$ and let $S$ be a nonexpansive mapping of $E$ into itself such that $F(S) \cap$ $\mathrm{EP}(F, D) \neq \emptyset$. Suppose $x_{1}=\sigma \in E$ and let $\left\{u_{n}\right\},\left\{y_{n}\right\}$, and $\left\{x_{n}\right\}$ by sequences generated by

$$
\begin{gathered}
F\left(u_{n}, y\right)+\left\langle D x_{n}, y-u_{n}\right\rangle+\frac{1}{r_{n}}\left\langle y-u_{n}, u_{n}-x_{n}\right\rangle \geq 0, \quad \forall y \in C, \\
y_{n}=\alpha_{n} \sigma+\left(1-\alpha_{n}\right) u_{n}, \\
x_{n+1}=\beta_{n} x_{n}+\left(1-\beta_{n}\right) S y_{n},
\end{gathered}
$$

where $\left\{\alpha_{n}\right\} \subset[0,1],\left\{\beta_{n}\right\} \subset[0,1]$, and $\left\{r_{n}\right\} \subset[0,2 \eta]$ satisfy some parameters controlling conditions. They proved that the sequence $\left\{x_{n}\right\}$ defined by (1.15) converges strongly to a common element of $F(S) \cap \mathrm{EF}(F, D)$.

On the other hand, iterative methods for nonexpansive mappings have recently been applied to solve convex minimization problems; see, for example, [32-35] and the 
references therein. Convex minimization problems have a great impact and influence in the development of almost all branches of pure and applied sciences.

A typical problem is to minimize a quadratic function over the set of the fixed points a nonexpansive mapping in a real Hilbert space $H$ :

$$
\min _{x \in E}\left\{\frac{1}{2}\langle A x, x\rangle-\langle x, b\rangle\right\}
$$

where $E$ is the fixed point set of a nonexpansive mapping $S$ on $H$ and $b$ is a given point in $H$. Assume that $A$ is a strongly positive bounded linear operator on $H$; that is, there exists a constant $\bar{\gamma}>0$ such that

$$
\langle A x, x\rangle \geq \bar{\gamma}\|x\|^{2}, \quad \forall x \in H .
$$

In 2006, Marino and Xu [36] considered the following iterative method:

$$
x_{n+1}=\epsilon_{n} \gamma f\left(x_{n}\right)+\left(1-\epsilon_{n} A\right) S x_{n}, \quad \forall n \geq 0 .
$$

They proved that if the sequence $\left\{\epsilon_{n}\right\}$ of parameters satisfies appropriate conditions, then the sequence $\left\{x_{n}\right\}$ generated by (1.18) converges strongly to the unique of the variational inequality

$$
\langle(A-\gamma f) z, x-z\rangle \geq 0, \quad \forall x \in F(S),
$$

which is the optimality condition for the minimization problem

$$
\min _{x \in F(S)}\left\{\frac{1}{2}\langle A x, x\rangle-h(x)\right\}
$$

where $h$ is a potential function for $\gamma f$ (i.e., $h^{\prime}(x)=\gamma f(x)$ for $x \in H$ ).

In 2008, Qin et al. [26] proposed the following iterative algorithm:

$$
\begin{gathered}
F\left(u_{n}, y\right)+\frac{1}{r_{n}}\left\langle y-u_{n}, u_{n}-x_{n}\right\rangle \geq 0, \quad \forall y \in H \\
x_{n+1}=\epsilon_{n} \gamma f\left(x_{n}\right)+\left(I-\epsilon_{n} A\right) \operatorname{SP}_{E}\left(I-\tau_{n} B\right) u_{n}
\end{gathered}
$$

where $A$ is a strongly positive linear bounded operator and $B$ is a relaxed cocoercive mapping of $E$ into $H$. They prove that if the sequences $\left\{\epsilon_{n}\right\},\left\{\tau_{n}\right\}$, and $\left\{r_{n}\right\}$ of parameters satisfy appropriate condition, then the sequences $\left\{x_{n}\right\}$ and $\left\{u_{n}\right\}$ both converge to the unique solution $z$ of the variational inequality

$$
\langle(A-\gamma f) z, x-z\rangle \geq 0, \quad \forall x \in F(S) \cap \operatorname{VI}(E, B) \cap \mathrm{EP}(F),
$$


which is the optimality condition for the minimization problem

$$
\min _{x \in F(S) \cap V I(E, B) \cap E P(F)}\left\{\frac{1}{2}\langle A x, x\rangle-h(x)\right\}
$$

where $h$ is a potential function for $\gamma f$ (i.e., $h^{\prime}(x)=\gamma f(x)$ for $x \in H$ ).

Furthermore, for finding approximate common fixed points of an infinite family of nonexpansive mappings $\left\{T_{n}\right\}$ under very mild conditions on the parameters, we need the following definition.

Definition 1.2 (see [37]). Let $\left\{T_{n}\right\}_{n=1}^{\infty}$ be a sequence of nonexpansive mappings of $E$ into itself and let $\left\{\mu_{n}\right\}_{n=1}^{\infty}$ be a sequence of nonnegative numbers in $[0,1]$. For each $n \geq 1$, define a mapping $W_{n}$ of $E$ into itself as follows:

$$
\begin{gathered}
U_{n, n+1}=I, \\
U_{n, n}=\mu_{n} T_{n} U_{n, n+1}+\left(1-\mu_{n}\right) I, \\
U_{n, n-1}=\mu_{n-1} T_{n-1} U_{n, n}+\left(1-\mu_{n-1}\right) I, \\
\vdots \\
U_{n, k}=\mu_{k} T_{k} U_{n, k+1}+\left(1-\mu_{k}\right) I, \\
U_{n, k-1}=\mu_{k-1} T_{k-1} U_{n, k}+\left(1-\mu_{k-1}\right) I, \\
\vdots \\
U_{n, 2}=\mu_{2} T_{2} U_{n, 3}+\left(1-\mu_{2}\right) I, \\
W_{n}=U_{n, 1}=\mu_{1} T_{1} U_{n, 2}+\left(1-\mu_{1}\right) I .
\end{gathered}
$$

Such a mappings $W_{n}$ is called the $W$-mapping generated by $T_{1}, T_{2}, \ldots, T_{n}$ and $\mu_{1}, \mu_{2}, \ldots, \mu_{n}$. It is obvious that $W_{n}$ is nonexpansive, and if $x=T_{n} x$, then $x=U_{n, k}=W_{n} x$.

On the other hand, Yao et al. [38] introduced and considered an iterative scheme for finding a common element of the set of solutions of the equilibrium problem and the set of common fixed points of an infinite family of nonexpansive mappings on $E$. Starting with an arbitrary initial $x_{1} \in H$, define sequences $\left\{x_{n}\right\}$ and $\left\{u_{n}\right\}$ recursively by

$$
\begin{gathered}
F\left(u_{n}, y\right)+\frac{1}{r_{n}}\left\langle y-u_{n}, u_{n}-x_{n}\right\rangle \geq 0, \quad \forall y \in H, \\
x_{n+1}=\epsilon_{n} \gamma f\left(x_{n}\right)+\beta_{n} x_{n}+\left(\left(1-\beta_{n}\right) I-\epsilon_{n} A\right) W_{n} u_{n}, \quad \forall n \geq 1,
\end{gathered}
$$

where $\left\{\epsilon_{n}\right\}$ is a sequence in $(0,1)$. It is proved [38] that under certain appropriate conditions imposed on $\left\{\epsilon_{n}\right\}$ and $\left\{r_{n}\right\}$, the sequence $\left\{x_{n}\right\}$ generated by (1.25) converges strongly to $z=P_{\bigcap_{n=1}^{\infty} F\left(T_{n}\right) \cap E \mathrm{EP}(F)}(I-A+\gamma f) z$. Very recently, Qin et al. [6] introduced an iterative scheme for finding a common fixed points of a finite family of nonexpansive mappings, the set of 
solutions of the variational inequality problem for a relaxed cocoercive mapping, and the set of solutions of the equilibrium problems in a real Hilbert space. Starting with an arbitrary initial $x_{1} \in H$, define sequences $\left\{x_{n}\right\}$ and $\left\{u_{n}\right\}$ recursively by

$$
\begin{gathered}
F\left(u_{n}, y\right)+\frac{1}{r_{n}}\left\langle y-u_{n}, u_{n}-x_{n}\right\rangle \geq 0, \quad \forall y \in H, \\
x_{n+1}=\epsilon_{n} \gamma f\left(W_{n} x_{n}\right)+\left(I-\epsilon_{n} A\right) W_{n} P_{E}\left(I-\tau_{n} B\right) u_{n}, \quad \forall n \geq 1,
\end{gathered}
$$

where $B$ is a relaxed $(u, v)$-cocoercive mapping and $A$ is a strongly positive linear bounded operator. They proved that under certain appropriate conditions imposed on $\left\{\epsilon_{n}\right\},\left\{\tau_{n}\right\}$, and $\left\{r_{n}\right\}$, the sequences $\left\{x_{n}\right\}$ and $\left\{u_{n}\right\}$ generated by (1.26) converge strongly to some point $z \in$ $\bigcap_{n=1}^{\infty} F\left(T_{n}\right) \cap \mathrm{EP}(F) \cap \mathrm{VI}(E, B)$, which is a unique solution of the variation inequality:

$$
\langle(A-\gamma f) z, x-z\rangle \geq 0, \quad \forall x \in \bigcap_{n=1}^{\infty} F\left(T_{n}\right) \cap \mathrm{EP}(F) \cap \mathrm{VI}(E, B)
$$

and is also the optimality for some minimization problems.

In this paper, motivated by iterative schemes considered in (1.15), (1.25), and (1.26) we will introduce a new iterative process (3.4) below for finding a common element of the set of fixed points of an infinite family of nonexpansive mappings, the set of solutions of the generalized equilibrium problem, and the set of solutions of variational inequality problem for a relaxed $(u, v)$-cocoercive mapping in a real Hilbert space. The results obtained in this paper improve and extend the recent ones announced by Yao et al. [38], S. Takahashi and W. Takahashi [27], and Qin et al. [6] and many others.

\section{Preliminaries}

Let $H$ be a real Hilbert space with inner product $\langle\cdot, \cdot\rangle$ and norm $\|\cdot\|$. Let $E$ be a nonempty closed convex subset of $H$. We denote weak convergence and strong convergence by notations $\rightarrow$ and $\rightarrow$, respectively. Recall that the (nearest point) projection $P_{E}$ from $H$ to $E$ assigns each $x \in H$ the unique point in $P_{E} x \in E$ satisfying the property

$$
\left\|x-P_{E} x\right\|=\min _{y \in E}\|x-y\| .
$$

The following characterizes the projection $P_{E}$.

We need some facts tools in a real Hilbert space $H$ which are listed as follows.

Lemma 2.1. For any $x \in H, z \in E$,

$$
z=P_{E} x \Longleftrightarrow\langle x-z, z-y\rangle \geq 0, \quad \forall y \in E
$$

It is well known that $P_{E}$ is a firmly nonexpansive mapping of $H$ onto $E$ and satisfies

$$
\left\|P_{E} x-P_{E} y\right\|^{2} \leq\left\langle P_{E} x-P_{E} y, x-y\right\rangle, \quad \forall x, y \in H .
$$


Moreover, $P_{E} x$ is characterized by the following properties: $P_{E} x \in E$ and for all $x \in H, y \in E$,

$$
\left\langle x-P_{E} x, y-P_{E} x\right\rangle \leq 0
$$

Lemma 2.2 (see [39]). Let $H$ be a Hilbert space, let $E$ be a nonempty closed convex subset of $H$, and let $B$ be a mapping of $E$ into $H$. Let $p \in E$. Then for $\lambda>0$,

$$
p \in \mathrm{VI}(E, B) \Longleftrightarrow p=P_{E}(p-\lambda B p)
$$

where $P_{E}$ is the metric projection of $H$ onto $E$.

It is clear from Lemma 2.2 that variational inequality and fixed point problem are equivalent. This alternative equivalent formulation has played a significant role in the studies of the variational inequalities and related optimization problems.

Lemma 2.3 (see [40]). Each Hilbert space $H$ satisfies Opials condition; that is, for any sequence $\left\{x_{n}\right\} \subset H$ with $x_{n} \rightarrow x$, the inequality

$$
\liminf _{n \rightarrow \infty}\left\|x_{n}-x\right\|<\liminf _{n \rightarrow \infty}\left\|x_{n}-y\right\|
$$

holds for each $y \in H$ with $y \neq x$.

Lemma 2.4 (see [36]). Assume that $A$ is a strongly positive linear bounded operator on $H$ with coefficient $\bar{\gamma}>0$ and $0<\rho \leq\|A\|^{-1}$. Then $\|I-\rho A\| \leq 1-\rho \bar{\gamma}$.

For solving the equilibrium problem for a bifunction $F: E \times E \rightarrow \mathbb{R}$, let us assume that $F$ satisfies the following conditions:

(A1) $F(x, x)=0$, for all $x \in E$;

(A2) $F$ is monotone, that is, $F(x, y)+F(y, x) \leq 0$, for all $x, y \in E$;

(A3) $\lim _{t \downarrow 0} F(t z+(1-t) x, y) \leq F(x, y)$, for all $x, y, z \in E$;

(A4) for each $x \in E, y \mapsto F(x, y)$ is convex and lower semicontinuous.

The following lemma appears implicitly in [4].

Lemma 2.5 (see [4]). Let $E$ be a nonempty closed convex subset of $H$ and let $F$ be a bifunction of $E \times E$ into $\mathbb{R}$ satisfying $(A 1)-(A 4)$. Let $r>0$ and $x \in H$. Then, there exists $z \in E$ such that

$$
F(z, y)+\frac{1}{r}\langle y-z, z-x\rangle \geq 0, \quad \forall y \in E
$$

The following lemma was also given in [5]. 
Lemma 2.6 (see [5]). Assume that $F: E \times E \rightarrow \mathbb{R}$ satisfies (A1)-(A4). For $r>0$ and $x \in H$, define a mapping $T_{r}: H \rightarrow E$ as follows:

$$
T_{r}(x)=\left\{z \in E: F(z, y)+\frac{1}{r}\langle y-z, z-x\rangle \geq 0, \forall y \in E\right\}
$$

for all $z \in H$. Then, the following holds:

(1) $T_{r}$ is single-valued;

(2) $T_{r}$ is firmly nonexpansive, that is, for any $x, y \in H$,

$$
\left\|T_{r} x-T_{r} y\right\|^{2} \leq\left\langle T_{r} x-T_{r} y, x-y\right\rangle
$$

(3) $F\left(T_{r}\right)=\operatorname{EP}(F)$;

(4) $\mathrm{EP}(F)$ is closed and convex.

Remark 2.7. Replacing $x$ with $x-r D x \in H$ in (2.7), then there exists $z \in E$, such that

$$
F(z, y)+\langle D x, y-z\rangle+\frac{1}{r}\langle y-z, z-x\rangle \geq 0, \quad \forall y \in E .
$$

Lemma 2.8 (see [41]). Let E be a nonempty closed convex subset of a real Hilbert space H. Let $T_{1}, T_{2}, \ldots$ be nonexpansive mappings of $E$ into itself such that $\bigcap_{n=1}^{\infty} F\left(T_{n}\right)$ is nonempty, and let $\mu_{1}, \mu_{2}, \ldots$ be real numbers such that $0 \leq \mu_{n} \leq b<1$ for every $n \geq 1$. Then, for every $x \in E$ and $k \in \mathbb{N}$, the limit $\lim _{n \rightarrow \infty} U_{n, k} x$ exists.

Using Lemma 2.8, one can define a mapping $W$ of $E$ into itself as follows:

$$
W x=\lim _{n \rightarrow \infty} W_{n} x=\lim _{n \rightarrow \infty} U_{n, 1} x,
$$

for every $x \in E$. Such a $W$ is called the $W$-mapping generated by $T_{1}, T_{2}, \ldots$ and $\mu_{1}, \mu_{2}, \ldots$ Throughout this paper, we will assume that $0 \leq \mu_{n} \leq b<1$ for every $n \geq 1$. Then, we have the following results.

Lemma 2.9 (see [41]). Let E be a nonempty closed convex subset of a real Hilbert space $H$. Let $T_{1}, T_{2}, \ldots$ be nonexpansive mappings of $E$ into itself such that $\bigcap_{n=1}^{\infty} F\left(T_{n}\right)$ is nonempty, let $\mu_{1}, \mu_{2}, \ldots$ be real numbers such that $0 \leq \mu_{n} \leq b<1$ for every $n \geq 1$. Then, $F(W)=\bigcap_{n=1}^{\infty} F\left(T_{n}\right)$.

Lemma 2.10 (see [7]). If $\left\{x_{n}\right\}$ is a bounded sequence in $E$, then $\lim _{n \rightarrow \infty}\left\|W x_{n}-W_{n} x_{n}\right\|=0$.

Lemma 2.11 (see [42]). Let $\left\{x_{n}\right\}$ and $\left\{z_{n}\right\}$ be bounded sequences in a Banach space $X$ and let $\left\{\beta_{n}\right\}$ be a sequence in $[0,1]$ with $0<\liminf _{n \rightarrow \infty} \beta_{n} \leq \limsup _{n \rightarrow \infty} \beta_{n}<1$. Suppose $x_{n+1}=\left(1-\beta_{n}\right) z_{n}+\beta_{n} x_{n}$ for all integers $n \geq 0$ and $\lim \sup _{n \rightarrow \infty}\left(\left\|z_{n+1}-z_{n}\right\|-\left\|x_{n+1}-x_{n}\right\|\right) \leq 0$. Then, $\lim _{n \rightarrow \infty}\left\|z_{n}-x_{n}\right\|=0$.

Lemma 2.12. Let $H$ be a real Hilbert space. Then the following inequality holds:

(1) $\|x+y\|^{2} \leq\|x\|^{2}+2\langle y, x+y\rangle$,

(2) $\|x+y\|^{2} \geq\|x\|^{2}+2\langle y, x\rangle$

for all $x, y \in H$. 
Lemma 2.13 (see [43]). Assume that $\left\{a_{n}\right\}$ is a sequence of nonnegative real numbers such that

$$
a_{n+1} \leq\left(1-l_{n}\right) a_{n}+\sigma_{n}, \quad \forall n \geq 0,
$$

where $\left\{l_{n}\right\}$ is a sequence in $(0,1)$ and $\left\{\sigma_{n}\right\}$ is a sequence in $\mathbb{R}$ such that

(1) $\sum_{n=1}^{\infty} l_{n}=\infty$,

(2) $\lim \sup _{n \rightarrow \infty}\left(\sigma_{n} / l_{n}\right) \leq 0$ or $\sum_{n=1}^{\infty}\left|\sigma_{n}\right|<\infty$.

Then $\lim _{n \rightarrow \infty} a_{n}=0$.

\section{Main Results}

In this section, we prove a strong convergence theorem of a new iterative method (3.4) for an infinite family of nonexpansive mappings and relaxed $(u, v)$-cocoercive mappings in a real Hilbert space.

We first prove the following lemmas.

Lemma 3.1. Let $H$ be a real Hilbert space, let $E$ be a nonempty closed convex subset of $H$, and let $D: E \rightarrow H$ be $\eta$-inverse-strongly monotone. It $0 \leq r_{n} \leq 2 \eta$, then $I-r_{n} D$ is a nonexpansive mapping in $H$.

Proof. For all $x, y \in E$ and $0 \leq r_{n} \leq 2 \eta$, we have

$$
\begin{aligned}
\left\|\left(I-r_{n} D\right) x-\left(I-r_{n} D\right) y\right\|^{2} & =\left\|(x-y)-r_{n}(D x-D y)\right\|^{2} \\
& =\|x-y\|^{2}-2 r_{n}\langle x-y, D x-D y\rangle+r_{n}^{2}\|D x-D y\|^{2} \\
& \leq\|x-y\|^{2}-2 r_{n} \eta\|D x-D y\|+r_{n}^{2}\|D x-D y\|^{2} \\
& =\|x-y\|^{2}+r_{n}\left(r_{n}-2 \eta\right)\|D x-D y\|^{2} \\
& \leq\|x-y\|^{2} .
\end{aligned}
$$

So, $I-r_{n} D$ is a nonexpansive mapping of $E$ into $H$.

Lemma 3.2. Let $H$ be a real Hilbert space, let $E$ be a nonempty closed convex subset of $H$, and let $B: E \rightarrow H$ be a relaxed $(u, v)$-cocoercive and $\xi$-Lipschitz continuous. If $0 \leq \tau_{n} \leq 2\left(v-u \xi^{2}\right) / \xi^{2}$, $v>u \xi^{2}$, then $I-\tau_{n} B$ is a nonexpansive mapping in $H$.

Proof. For any $x, y \in E$ and $\tau_{n} \leq 2\left(v-u \xi^{2}\right) / \xi^{2}, v>u \xi^{2}$.

Putting $r=1+2 \tau_{n} u \xi^{2}-2 \tau_{n} v+\tau_{n}^{2} \xi^{2}$, we obtain

$$
\begin{aligned}
\tau_{n} \leq \frac{2\left(v-u \xi^{2}\right)}{\xi^{2}} & \Longleftrightarrow \tau_{n} \xi^{2}+2 u \xi^{2}-2 v \leq 0 \\
& \Longleftrightarrow \tau_{n}^{2} \xi^{2}+2 \tau_{n} u \xi^{2}-2 \tau_{n} v \leq 0 \\
& \Longleftrightarrow 1+\tau_{n}^{2} \xi^{2}+2 \tau_{n} u \xi^{2}-2 \tau_{n} v \leq 1
\end{aligned}
$$


that is, $r \leq 1$. It follows that

$$
\begin{aligned}
\left\|\left(I-\tau_{n} B\right) x-\left(I-\tau_{n} B\right) y\right\|^{2} & =\left\|(x-y)-\tau_{n}(B x-B y)\right\|^{2} \\
& =\|x-y\|^{2}-2 \tau_{n}\langle x-y, B x-B y\rangle+\tau_{n}^{2}\|B x-B y\|^{2} \\
& \leq\|x-y\|^{2}-2 \tau_{n}\left\{-u\|B x-B y\|^{2}+v\|x-y\|^{2}\right\}+\tau_{n}^{2}\|B x-B y\|^{2} \\
& \leq\|x-y\|^{2}+2 \tau_{n} u \xi^{2}\|x-y\|^{2}-2 \tau_{n} v\|x-y\|^{2}+\tau_{n}^{2} \xi^{2}\|x-y\|^{2} \\
& =\left(1+2 \tau_{n} u \xi^{2}-2 \tau_{n} v+\tau_{n}^{2} \xi^{2}\right)\|x-y\|^{2} \\
& =r\|x-y\|^{2} \\
& \leq\|x-y\|^{2},
\end{aligned}
$$

for all $x, y \in E$. Thus $\left\|\left(I-\tau_{n} B\right) x-\left(I-\tau_{n} B\right) y\right\| \leq\|x-y\|$.

So, $I-\tau_{n} B$ is a nonexpansive mapping of $E$ into $H$.

Now, we prove the following main theorem.

Theorem 3.3. Let $E$ be a nonempty closed convex subset of a real Hilbert space $H$, and let $F: E \times E \rightarrow$ $\mathbb{R}$ be a bifunction satisfying (A1)-(A4). Let

(1) $\left\{T_{n}\right\}$ be an infinite family of nonexpansive mappings of $E$ into $E$;

(2) $D$ be an $\eta$-inverse strongly monotone mappings of $E$ into $H$;

(3) B be relaxed $(u, v)$-cocoercive and $\xi$-Lipschitz continuous mappings of $E$ into $H$.

Assume that $\Theta:=\bigcap_{n=1}^{\infty} F\left(T_{n}\right) \cap \mathrm{EP}(F, D) \cap \operatorname{VI}(E, B) \neq \emptyset$. Let $f: E \rightarrow E$ be a contraction mapping with $0<\alpha<1$ and let $A$ be a strongly positive linear bounded operator on $H$ with coefficient $\bar{\gamma}>0$ and $0<\gamma<\bar{\gamma} / \alpha$. Let $\left\{x_{n}\right\},\left\{y_{n}\right\},\left\{k_{n}\right\}$, and $\left\{u_{n}\right\}$ be sequences generated by

$$
\begin{gathered}
x_{1} \in E \text { chosen arbitrary, } \\
F\left(u_{n}, y\right)+\left\langle D x_{n}, y-u_{n}\right\rangle+\frac{1}{r_{n}}\left\langle y-u_{n}, u_{n}-x_{n}\right\rangle \geq 0, \quad \forall y \in E, \\
y_{n}=\varphi_{n} u_{n}+\left(1-\varphi_{n}\right) W_{n} P_{E}\left(u_{n}-\delta_{n} B u_{n}\right), \\
k_{n}=\alpha_{n} x_{n}+\left(1-\alpha_{n}\right) W_{n} P_{E}\left(y_{n}-\lambda_{n} B y_{n}\right), \\
x_{n+1}=\epsilon_{n} \gamma f\left(W_{n} x_{n}\right)+\beta_{n} x_{n}+\left(\left(1-\beta_{n}\right) I-\epsilon_{n} A\right) W_{n} P_{E}\left(k_{n}-\tau_{n} B k_{n}\right), \quad \forall n \geq 1,
\end{gathered}
$$

where $\left\{W_{n}\right\}$ is the sequence generated by (1.24) and $\left\{\epsilon_{n}\right\},\left\{\alpha_{n}\right\},\left\{\varphi_{n}\right\}$, and $\left\{\beta_{n}\right\}$ are sequences in $(0,1)$ satisfy the following conditions:

(C1) $\lim _{n \rightarrow \infty} \epsilon_{n}=0, \sum_{n=1}^{\infty} \epsilon_{n}=\infty$,

(C2) $\lim _{n \rightarrow \infty} \alpha_{n}=\lim _{n \rightarrow \infty} \varphi_{n}=0$, 
(C3) $0<\liminf _{n \rightarrow \infty} \beta_{n} \leq \lim \sup _{n \rightarrow \infty} \beta_{n}<1$,

(C4) $\liminf _{n \rightarrow \infty} r_{n}>0$ and $\lim _{n \rightarrow \infty}\left|r_{n+1}-r_{n}\right|=0$,

(C5) $\lim _{n \rightarrow \infty}\left|\lambda_{n+1}-\lambda_{n}\right|=\lim _{n \rightarrow \infty}\left|\delta_{n+1}-\delta_{n}\right|=\lim _{n \rightarrow \infty}\left|\tau_{n+1}-\tau_{n}\right|=0$,

(C6) $\left\{\tau_{n}\right\},\left\{\lambda_{n}\right\},\left\{\delta_{n}\right\} \subset[a, b]$ for some $a, b$ with $0 \leq a \leq b \leq 2\left(v-u \xi^{2}\right) / \xi^{2}, v>u \xi^{2}$,

(C7) $\left\{r_{n}\right\} \subset[c, d]$ for some $c, d$ with $0<c<d<2 \eta$.

Then, $\left\{x_{n}\right\}$ and $\left\{u_{n}\right\}$ converge strongly to a point $z \in \Theta$, where $z=P_{\Theta}(I-A+\gamma f)(z)$, which solves the variational inequality

$$
\langle(A-\gamma f) z, x-z\rangle \geq 0, \quad \forall x \in \Theta,
$$

which is the optimality condition fot the minimization problem

$$
\min _{x \in \Theta}\left\{\frac{1}{2}\langle A x, x\rangle-h(x)\right\}
$$

where $h$ is a potential function for $\gamma f$ (i.e., $h^{\prime}(x)=\gamma f(x)$ for $x \in H$ ).

Proof. Since $\lim _{n \rightarrow \infty} \epsilon_{n}=0$ by the condition (C1) and limsup $\sup _{n \rightarrow \infty} \beta_{n}<1$, we may assume, without loss of generality, that $\epsilon_{n} \leq\left(1-\beta_{n}\right)\|A\|^{-1}$. Since $A$ is a strongly positive bounded linear operator on $H$, then

$$
\|A\|=\sup \{|\langle A x, x\rangle|: x \in H,\|x\|=1\} .
$$

Observe that

$$
\begin{aligned}
\left\langle\left(\left(1-\beta_{n}\right) I-\epsilon_{n} A\right) x, x\right\rangle & =1-\beta_{n}-\epsilon_{n}\langle A x, x\rangle \\
& \geq 1-\beta_{n}-\epsilon_{n}\|A\| \\
& \geq 0,
\end{aligned}
$$

that is to say $\left(1-\beta_{n}\right) I-\epsilon_{n} A$ is positive. It follows that

$$
\begin{aligned}
\left\|\left(1-\beta_{n}\right) I-\epsilon_{n} A\right\| & =\sup \left\{\left|\left\langle\left(\left(1-\beta_{n}\right) I-\epsilon_{n} A\right) x, x\right\rangle\right|: x \in H,\|x\|=1\right\} \\
& =\sup \left\{1-\beta_{n}-\epsilon_{n}\langle A x, x\rangle: x \in H,\|x\|=1\right\} \\
& \leq 1-\beta_{n}-\epsilon_{n} \bar{\gamma} .
\end{aligned}
$$

We will divide the proof of Theorem 3.3 into six steps.

Step 1. We prove that there exists $z \in E$ such that $z=P_{\bigcap_{n=1}^{\infty} F\left(T_{n}\right) \cap \operatorname{EP}(F, D) \cap V I(E, B)}(I-A+\gamma f) z$. 
Let $\mathfrak{I}=P_{\bigcap_{n=1}^{\infty} F\left(T_{n}\right) \cap E P(F, D) \cap V I(E, B)}$. Note that $f$ is a contraction mapping of $E$ into itself with coefficient $\alpha \in(0,1)$. Then, we have

$$
\begin{aligned}
\|\Im(I-A+\gamma f)(x)-\Im(I-A+\gamma f)(y)\| & \leq\|(I-A+\gamma f)(x)-(I-A+\gamma f)(y)\| \\
& \leq\|I-A\|\|x-y\|+\gamma\|f(x)-f(y)\| \\
& \leq(1-\bar{\gamma})\|x-y\|+\gamma \alpha\|x-y\| \\
& =(1-(\bar{\gamma}-\alpha \gamma))\|x-y\|, \quad \forall x, y \in H .
\end{aligned}
$$

Therefore, $\Im(I-A+\gamma f)$ is a contraction mapping of $E$ into itself. Therefore by the Banach Contraction Mapping Principle guarantee that $\Im(I-A+\gamma f)$ has a unique fixed point, say $z \in E$. That is, $z=\mathfrak{I}(I-A+\gamma f)(z)=P_{\bigcap_{n=1}^{\infty} F\left(T_{n}\right) \cap E P(F, D) \cap V I(E, B)}(I-A+\gamma f)(z)$.

Step 2. We prove that $\left\{x_{n}\right\}$ is bounded.

Since

$$
F\left(u_{n}, y\right)+\left\langle D x_{n}, y-u_{n}\right\rangle+\frac{1}{r_{n}}\left\langle y-u_{n}, u_{n}-x_{n}\right\rangle \geq 0, \quad \forall y \in E,
$$

we obtain

$$
F\left(u_{n}, y\right)+\frac{1}{r_{n}}\left\langle y-u_{n}, u_{n}-\left(I-r_{n} D\right) x_{n}\right\rangle \geq 0, \quad \forall y \in E
$$

From Lemma 2.6, we have $u_{n}=T_{r_{n}}\left(x_{n}-r_{n} D x_{n}\right)$, for all $n \in \mathbb{N}$.

For any $p \in \Theta:=\bigcap_{n=1}^{\infty} F\left(T_{n}\right) \cap \operatorname{EP}(F, D) \cap \operatorname{VI}(E, B)$, it follows from $p \in \operatorname{EP}(F, D)$ that

$$
F(p, y)+\langle y-p, D p\rangle \geq 0, \quad \forall y \in E
$$

So, we have

$$
F(p, y)+\frac{1}{r_{n}}\left\langle y-p, p-\left(p-r_{n} D p\right)\right\rangle \geq 0, \quad \forall y \in E .
$$

By Lemma 2.6 again, we have $p=T_{r_{n}}\left(p-r_{n} D p\right)$, for all $n \in \mathbb{N}$. If follows that

$$
\begin{aligned}
\left\|u_{n}-p\right\| & =\left\|T_{r_{n}}\left(x_{n}-r_{n} D x_{n}\right)-T_{r_{n}}\left(p-r_{n} D p\right)\right\| \\
& \leq\left\|\left(x_{n}-r_{n} D x_{n}\right)-\left(p-r_{n} D p\right)\right\| \\
& =\left\|\left(I-r_{n} D\right) x_{n}-\left(I-r_{n} D\right) p\right\| \leq\left\|x_{n}-p\right\| .
\end{aligned}
$$


If we applied Lemma 3.2, we get $I-\lambda_{n} B$ and $I-\delta_{n} B$ are nonexpansive. Since $p \in \operatorname{VI}(E, B)$ and $W_{n}$ is a nonexpansive, we have $p=W_{n} P_{E}\left(p-\lambda_{n} B p\right)=W_{n} P_{E}\left(p-\delta_{n} B p\right)$, and we have

$$
\begin{aligned}
\left\|y_{n}-p\right\| & \leq \varphi_{n}\left\|u_{n}-p\right\|+\left(1-\varphi_{n}\right)\left\|W_{n} P_{E}\left(u_{n}-\delta_{n} B u_{n}\right)-W_{n} P_{E}\left(p-\delta_{n} B p\right)\right\| \\
& \leq \varphi_{n}\left\|u_{n}-p\right\|+\left(1-\varphi_{n}\right)\left\|\left(u_{n}-\delta_{n} B u_{n}\right)-\left(p-\delta_{n} B p\right)\right\| \\
& =\varphi_{n}\left\|u_{n}-p\right\|+\left(1-\varphi_{n}\right)\left\|\left(I-\delta_{n} B\right) u_{n}-\left(I-\delta_{n} B\right) p\right\| \\
& \leq \varphi_{n}\left\|u_{n}-p\right\|+\left(1-\varphi_{n}\right)\left\|u_{n}-p\right\| \\
& \leq\left\|u_{n}-p\right\| \leq\left\|x_{n}-p\right\| .
\end{aligned}
$$

It follows that

$$
\begin{aligned}
\left\|k_{n}-p\right\| & \leq \alpha_{n}\left\|x_{n}-p\right\|+\left(1-\alpha_{n}\right)\left\|W_{n} P_{E}\left(y_{n}-\lambda_{n} B y_{n}\right)-W_{n} P_{E}\left(p-\lambda_{n} B p\right)\right\| \\
& \leq \alpha_{n}\left\|x_{n}-p\right\|+\left(1-\alpha_{n}\right)\left\|\left(y_{n}-\lambda_{n} B y_{n}\right)-\left(p-\lambda_{n} B p\right)\right\| \\
& =\alpha_{n}\left\|x_{n}-p\right\|+\left(1-\alpha_{n}\right)\left\|\left(I-\lambda_{n} B\right) y_{n}-\left(I-\lambda_{n} B\right) p\right\| \\
& \leq \alpha_{n}\left\|x_{n}-p\right\|+\left(1-\alpha_{n}\right)\left\|y_{n}-p\right\| \\
& \leq \alpha_{n}\left\|x_{n}-p\right\|+\left(1-\alpha_{n}\right)\left\|x_{n}-p\right\|=\left\|x_{n}-p\right\|
\end{aligned}
$$

which yields that

$$
\begin{aligned}
\left\|x_{n+1}-p\right\| & =\left\|\epsilon_{n}\left(\gamma f\left(x_{n}\right)-A p\right)+\beta_{n}\left(x_{n}-p\right)+\left(\left(1-\beta_{n}\right) I-\epsilon_{n} A\right)\left(W_{n} P_{E}\left(k_{n}-\tau_{n} B k_{n}\right)-p\right)\right\| \\
& \leq\left(1-\beta_{n}-\epsilon_{n} \bar{\gamma}\right)\left\|P_{E}\left(I-\tau_{n} B\right) k_{n}-p\right\|+\beta_{n}\left\|x_{n}-p\right\|+\epsilon_{n}\left\|\gamma f\left(x_{n}\right)-A p\right\| \\
& \leq\left(1-\beta_{n}-\epsilon_{n} \bar{\gamma}\right)\left\|k_{n}-p\right\|+\beta_{n}\left\|x_{n}-p\right\|+\epsilon_{n}\left\|\gamma f\left(x_{n}\right)-A p\right\| \\
& \leq\left(1-\beta_{n}-\epsilon_{n} \bar{\gamma}\right)\left\|x_{n}-p\right\|+\beta_{n}\left\|x_{n}-p\right\|+\epsilon_{n}\left\|\gamma f\left(x_{n}\right)-A p\right\| \\
& \leq\left(1-\epsilon_{n} \bar{\gamma}\right)\left\|x_{n}-p\right\|+\epsilon_{n} \gamma\left\|f\left(x_{n}\right)-f(p)\right\|+\epsilon_{n}\|\gamma f(p)-A p\| \\
& \leq\left(1-\epsilon_{n} \bar{\gamma}\right)\left\|x_{n}-p\right\|+\epsilon_{n} \gamma \alpha\left\|x_{n}-p\right\|+\epsilon_{n}\|\gamma f(p)-A p\| \\
& =\left(1-(\bar{\gamma}-\alpha \gamma) \epsilon_{n}\right)\left\|x_{n}-p\right\|+(\bar{\gamma}-\alpha \gamma) \epsilon_{n} \frac{\|\gamma f(p)-A p\|}{\bar{\gamma}-\alpha \gamma} .
\end{aligned}
$$

This in turn implies that

$$
\left\|x_{n}-p\right\| \leq \max \left\{\left\|x_{1}-p\right\|, \frac{\|\gamma f(p)-A p\|}{\bar{\gamma}-\alpha \gamma}\right\}, \quad n \in \mathbb{N} .
$$


Therefore, $\left\{x_{n}\right\}$ is bounded. We also obtain that $\left\{u_{n}\right\},\left\{k_{n}\right\},\left\{y_{n}\right\},\left\{B u_{n}\right\},\left\{B k_{n}\right\},\left\{B y_{n}\right\}$, $\left\{W_{n} u_{n}\right\},\left\{W_{n} k_{n}\right\},\left\{W_{n} y_{n}\right\}$, and $\left\{f\left(W_{n} x_{n}\right)\right\}$ are all bounded.

Step 3. We claim that $\lim _{n \rightarrow \infty}\left\|x_{n+1}-x_{n}\right\|=0$ and $\lim _{n \rightarrow \infty}\left\|W_{n} \theta_{n}-x_{n}\right\|=0$.

From Lemma 2.6, we have $u_{n}=T_{r_{n}}\left(x_{n}-r_{n} D x_{n}\right)$ and $u_{n+1}=T_{r_{n+1}}\left(x_{n+1}-r_{n+1} D x_{n+1}\right)$. Let $\varpi_{n}=x_{n}-r_{n} D x_{n}$, we get $u_{n}=T_{r_{n}} \varpi_{n}, u_{n+1}=T_{r_{n+1}} \varpi_{n+1}$, and so

$$
\begin{gathered}
F\left(u_{n}, y\right)+\frac{1}{r_{n}}\left\langle y-u_{n}, u_{n}-\varpi_{n}\right\rangle \geq 0, \quad \forall y \in E \\
F\left(u_{n+1}, y\right)+\frac{1}{r_{n+1}}\left\langle y-u_{n+1}, u_{n+1}-\varpi_{n+1}\right\rangle \geq 0, \quad \forall y \in E .
\end{gathered}
$$

Putting $y=u_{n+1}$ in (3.20) and $y=u_{n}$ in (3.21), we have

$$
\begin{gathered}
F\left(u_{n}, u_{n+1}\right)+\frac{1}{r_{n}}\left\langle u_{n+1}-u_{n}, u_{n}-\varpi_{n}\right\rangle \geq 0, \\
F\left(u_{n+1}, u_{n}\right)+\frac{1}{r_{n+1}}\left\langle u_{n}-u_{n+1}, u_{n+1}-\varpi_{n+1}\right\rangle \geq 0 .
\end{gathered}
$$

So, from the monotonicity of $F$, we get

$$
\left\langle u_{n+1}-u_{n}, \frac{u_{n}-\varpi_{n}}{r_{n}}-\frac{u_{n+1}-\varpi_{n+1}}{r_{n+1}}\right\rangle \geq 0
$$

and hence

$$
\left\langle u_{n+1}-u_{n}, u_{n}-u_{n+1}+u_{n+1}-\varpi_{n}-\frac{r_{n}}{r_{n+1}}\left(u_{n+1}-\varpi_{n+1}\right)\right\rangle \geq 0
$$

Without loss of generality, let us assume that there exists a real number $c$ such that $r_{n}>c>0$ for all $n \in \mathbb{N}$. Then, we have

$$
\begin{aligned}
\left\|u_{n+1}-u_{n}\right\|^{2} & \leq\left\langle u_{n+1}-u_{n}, \varpi_{n+1}-\varpi_{n}+\left(1-\frac{r_{n}}{r_{n+1}}\right)\left(u_{n+1}-\varpi_{n+1}\right)\right\rangle \\
& \leq\left\|u_{n+1}-u_{n}\right\|\left\{\left\|\varpi_{n+1}-\varpi_{n}\right\|+\left|1-\frac{r_{n}}{r_{n+1}}\right|\left\|u_{n+1}-\varpi_{n+1}\right\|\right\}
\end{aligned}
$$


and hence

$$
\begin{aligned}
\left\|u_{n+1}-u_{n}\right\| \leq & \left\|\varpi_{n+1}-\varpi_{n}\right\|+\frac{1}{c}\left|r_{n+1}-r_{n}\right|\left\|u_{n+1}-\varpi_{n+1}\right\| \\
= & \left\|x_{n+1}-r_{n+1} D x_{n+1}-\left(x_{n}-r_{n} D x_{n}\right)\right\|+\frac{1}{c}\left|r_{n+1}-r_{n}\right|\left\|u_{n+1}-\varpi_{n+1}\right\| \\
\leq & \left\|x_{n+1}-r_{n+1} D x_{n+1}-\left(x_{n}-r_{n+1} D x_{n}\right)\right\|+\left|r_{n+1}-r_{n}\right|\left\|D x_{n}\right\| \\
& +\frac{1}{c}\left|r_{n+1}-r_{n}\right|\left\|u_{n+1}-\varpi_{n+1}\right\| \\
\leq & \left\|x_{n+1}-x_{n}\right\|+\left|r_{n+1}-r_{n}\right|\left\|D x_{n}\right\|+\frac{1}{c}\left|r_{n+1}-r_{n}\right|\left\|u_{n+1}-\varpi_{n+1}\right\| \\
\leq & \left\|x_{n+1}-x_{n}\right\|+M_{1}\left|r_{n+1}-r_{n}\right|
\end{aligned}
$$

where $M_{1}=\sup \left\{\left\|D x_{n}\right\|+(1 / c)\left\|u_{n+1}-\varpi_{n+1}\right\|: n \in \mathbb{N}\right\}$.

Put $\theta_{n}=P_{E}\left(k_{n}-\tau_{n} B k_{n}\right), \phi_{n}=P_{E}\left(y_{n}-\lambda_{n} B y_{n}\right)$, and $\psi_{n}=P_{E}\left(u_{n}-\delta_{n} B u_{n}\right)$. Since $I-\tau_{n} B$, I $-\lambda_{n} B$, and $I-\delta_{n} B$ are nonexpansive, then we have the following some estimates:

$$
\begin{aligned}
\left\|\psi_{n+1}-\psi_{n}\right\| & \leq\left\|P_{E}\left(u_{n+1}-\delta_{n+1} B u_{n+1}\right)-P_{E}\left(u_{n}-\delta_{n} B u_{n}\right)\right\| \\
& \leq\left\|\left(u_{n+1}-\delta_{n+1} B u_{n+1}\right)-\left(u_{n}-\delta_{n} B u_{n}\right)\right\| \\
& =\left\|\left(u_{n+1}-\delta_{n+1} B u_{n+1}\right)-\left(u_{n}-\delta_{n+1} B u_{n}\right)+\left(\delta_{n}-\delta_{n+1}\right) B u_{n}\right\| \\
& \leq\left\|\left(u_{n+1}-\delta_{n+1} B u_{n+1}\right)-\left(u_{n}-\delta_{n+1} B u_{n}\right)\right\|+\left|\delta_{n}-\delta_{n+1}\right|\left\|B u_{n}\right\| \\
& =\left\|\left(I-\delta_{n+1} B\right) u_{n+1}-\left(I-\delta_{n+1} B\right) u_{n}\right\|+\left|\delta_{n}-\delta_{n+1}\right|\left\|B u_{n}\right\| \\
& \leq\left\|u_{n+1}-u_{n}\right\|+\left|\delta_{n}-\delta_{n+1}\right|\left\|B u_{n}\right\| .
\end{aligned}
$$

Similarly, we can prove that

$$
\begin{aligned}
\left\|\phi_{n+1}-\phi_{n}\right\| & \leq\left\|y_{n+1}-y_{n}\right\|+\left|\lambda_{n}-\lambda_{n+1}\right|\left\|B y_{n}\right\|, \\
\left\|\theta_{n+1}-\theta_{n}\right\| & \leq\left\|k_{n+1}-k_{n}\right\|+\left|\tau_{n}-\tau_{n+1}\right|\left\|B k_{n}\right\| .
\end{aligned}
$$

Since $T_{i}$ and $U_{n, i}$ are nonexpansive, we deduce that, for each $n \leq 1$,

$$
\begin{aligned}
\left\|W_{n+1} \psi_{n}-W_{n} \psi_{n}\right\| & =\left\|\mu_{1} T_{1} U_{n+1,2} \psi_{n}-\mu_{1} T_{1} U_{n, 2} \psi_{n}\right\| \\
& \leq \mu_{1}\left\|U_{n+1,2} \psi_{n}-U_{n, 2} \psi_{n}\right\| \\
& =\mu_{1}\left\|\mu_{2} T_{2} U_{n+1,3} \psi_{n}-\mu_{2} T_{2} U_{n, 3} \psi_{n}\right\| \\
& \leq \mu_{1} \mu_{2}\left\|U_{n+1,3} \psi_{n}-U_{n, 3} \psi_{n}\right\|
\end{aligned}
$$


Journal of Inequalities and Applications

$$
\begin{aligned}
& \vdots \\
& \leq \prod_{i=1}^{n} \mu_{i}\left\|U_{n+1, n+1} \psi_{n}-U_{n, n+1} \psi_{n}\right\| \\
& \leq M_{2} \prod_{i=1}^{n} \mu_{i}
\end{aligned}
$$

where $M_{2} \geq 0$ is a constant such that $\left\|U_{n+1, n+1} \psi_{n}-U_{n, n+1} \psi_{n}\right\| \leq M_{2}$ for all $n \geq 0$.

Similarly, we can obtain that there exist nonnegative numbers $M_{3}, M_{4}$ such that

$$
\left\|U_{n+1, n+1} \varphi_{n}-U_{n, n+1} \varphi_{n}\right\| \leq M_{3}, \quad\left\|U_{n+1, n+1} \theta_{n}-U_{n, n+1} \theta_{n}\right\| \leq M_{4}
$$

and so are

$$
\left\|W_{n+1} \phi_{n}-W_{n} \phi_{n}\right\| \leq M_{3} \prod_{i=1}^{n} \mu_{i}, \quad\left\|W_{n+1} \theta_{n}-W_{n} \theta_{n}\right\| \leq M_{4} \prod_{i=1}^{n} \mu_{i}
$$

Observing that

$$
\begin{gathered}
y_{n}=\varphi_{n} u_{n}+\left(1-\varphi_{n}\right) W_{n} \psi_{n}, \\
y_{n+1}=\varphi_{n+1} u_{n+1}+\left(1-\varphi_{n+1}\right) W_{n+1} \psi_{n+1},
\end{gathered}
$$

we obtain

$$
y_{n}-y_{n+1}=\varphi_{n}\left(u_{n}-u_{n+1}\right)+\left(1-\varphi_{n}\right)\left(W_{n} \psi_{n}-W_{n+1} \psi_{n+1}\right)+\left(W_{n+1} \psi_{n+1}-u_{n+1}\right)\left(\varphi_{n+1}-\varphi_{n}\right)
$$

which yields that

$$
\begin{aligned}
\left\|y_{n}-y_{n+1}\right\| \leq & \varphi_{n}\left\|u_{n}-u_{n+1}\right\|+\left(1-\varphi_{n}\right)\left\|W_{n+1} \psi_{n+1}-W_{n} \psi_{n}\right\|+\left|\varphi_{n+1}-\varphi_{n}\right|\left\|W_{n+1} \psi_{n+1}-u_{n+1}\right\| \\
\leq & \varphi_{n}\left\|u_{n}-u_{n+1}\right\|+\left(1-\varphi_{n}\right)\left\{\left\|W_{n+1} \psi_{n+1}-W_{n+1} \psi_{n}\right\|+\left\|W_{n+1} \psi_{n}-W_{n} \psi_{n}\right\|\right\} \\
& +\left|\varphi_{n+1}-\varphi_{n}\right|\left\|W_{n+1} \psi_{n+1}-u_{n+1}\right\| \\
\leq & \varphi_{n}\left\|u_{n}-u_{n+1}\right\|+\left(1-\varphi_{n}\right)\left\{\left\|\psi_{n+1}-\psi_{n}\right\|+\left\|W_{n+1} \psi_{n}-W_{n} \psi_{n}\right\|\right\} \\
& +\left|\varphi_{n+1}-\varphi_{n}\right|\left\|W_{n+1} \psi_{n+1}-u_{n+1}\right\| \\
\leq & \varphi_{n}\left\|u_{n}-u_{n+1}\right\|+\left(1-\varphi_{n}\right)\left\|\psi_{n+1}-\psi_{n}\right\|+\left\|W_{n+1} \psi_{n}-W_{n} \psi_{n}\right\| \\
& +\left|\varphi_{n+1}-\varphi_{n}\right|\left\|W_{n+1} \psi_{n+1}-u_{n+1}\right\| .
\end{aligned}
$$


Substitution of (3.27) and (3.30) into (3.35) yields that

$$
\begin{aligned}
\left\|y_{n}-y_{n+1}\right\| \leq & \varphi_{n}\left\|u_{n}-u_{n+1}\right\|+\left(1-\varphi_{n}\right)\left\{\left\|u_{n+1}-u_{n}\right\|+\left|\delta_{n}-\delta_{n+1}\right|\left\|B u_{n}\right\|\right\} \\
& +M_{2} \prod_{i=1}^{n} \mu_{i}+\left|\varphi_{n+1}-\varphi_{n}\right|\left\|W_{n+1} \psi_{n+1}-u_{n+1}\right\| \\
= & \left\|u_{n}-u_{n+1}\right\|+\left(1-\varphi_{n}\right)\left|\delta_{n}-\delta_{n+1}\right|\left\|B u_{n}\right\| \\
& +M_{2} \prod_{i=1}^{n} \mu_{i}+\left\|W_{n+1} \psi_{n+1}-u_{n+1}\right\|\left|\varphi_{n+1}-\varphi_{n}\right| \\
& \leq\left\|u_{n}-u_{n+1}\right\|+M_{5}\left(\left|\delta_{n}-\delta_{n+1}\right|+\left|\varphi_{n+1}-\varphi_{n}\right|\right)+M_{2} \prod_{i=1}^{n} \mu_{i}
\end{aligned}
$$

where $M_{5}$ is an appropriate constant such that $M_{5}=\max \left\{\sup _{n \geq 1}\left\|B u_{n}\right\|, \sup _{n \geq 1}\left\|W_{n} \psi_{n}-u_{n}\right\|\right\}$. Observing that

$$
\begin{gathered}
k_{n}=\alpha_{n} x_{n}+\left(1-\alpha_{n}\right) W_{n} \phi_{n}, \\
k_{n+1}=\alpha_{n+1} x_{n+1}+\left(1-\alpha_{n+1}\right) W_{n} \phi_{n+1},
\end{gathered}
$$

we obtain

$$
k_{n}-k_{n+1}=\alpha_{n}\left(x_{n}-x_{n+1}\right)+\left(1-\alpha_{n}\right)\left(W_{n} \phi_{n}-W_{n+1} \phi_{n+1}\right)+\left(W_{n+1} \phi_{n+1}-x_{n+1}\right)\left(\alpha_{n+1}-\alpha_{n}\right),
$$

which yields that

$$
\begin{aligned}
\left\|k_{n}-k_{n+1}\right\| \leq & \alpha_{n}\left\|x_{n}-x_{n+1}\right\|+\left(1-\alpha_{n}\right)\left\|W_{n} \phi_{n}-W_{n+1} \phi_{n+1}\right\|+\left|\alpha_{n+1}-\alpha_{n}\right|\left\|W_{n+1} \phi_{n+1}-x_{n+1}\right\| \\
\leq & \alpha_{n}\left\|x_{n}-x_{n+1}\right\|+\left(1-\alpha_{n}\right)\left\{\left\|W_{n+1} \phi_{n+1}-W_{n+1} \phi_{n}\right\|+\left\|W_{n+1} \phi_{n}-W_{n} \phi_{n}\right\|\right\} \\
& +\left|\alpha_{n+1}-\alpha_{n}\right|\left\|W_{n+1} \phi_{n+1}-x_{n+1}\right\| \\
\leq & \alpha_{n}\left\|x_{n}-x_{n+1}\right\|+\left(1-\alpha_{n}\right)\left\|\phi_{n+1}-\phi_{n}\right\|+\left\|W_{n+1} \phi_{n}-W_{n} \phi_{n}\right\| \\
& +\left|\alpha_{n+1}-\alpha_{n}\right|\left\|W_{n+1} \phi_{n+1}-x_{n+1}\right\| .
\end{aligned}
$$


Journal of Inequalities and Applications

Substitution of (3.28) and (3.32) into (3.39) yields that

$$
\begin{aligned}
\left\|k_{n}-k_{n+1}\right\| \leq & \alpha_{n}\left\|x_{n}-x_{n+1}\right\|+\left(1-\alpha_{n}\right)\left\{\left\|y_{n+1}-y_{n}\right\|+\left|\lambda_{n}-\lambda_{n+1}\right|\left\|B y_{n}\right\|\right\} \\
& +M_{3} \prod_{i=1}^{n} \mu_{i}+\left|\alpha_{n+1}-\alpha_{n}\right|\left\|W_{n+1} \phi_{n+1}-x_{n+1}\right\| \\
= & \alpha_{n}\left\|x_{n}-x_{n+1}\right\|+\left(1-\alpha_{n}\right)\left\|y_{n+1}-y_{n}\right\|+\left(1-\alpha_{n}\right)\left|\lambda_{n}-\lambda_{n+1}\right|\left\|B y_{n}\right\| \\
& +M_{3} \prod_{i=1}^{n} \mu_{i}+\left|\alpha_{n+1}-\alpha_{n}\right|\left\|W_{n+1} \phi_{n+1}-x_{n+1}\right\| \\
\leq & \alpha_{n}\left\|x_{n}-x_{n+1}\right\|+\left(1-\alpha_{n}\right)\left\|y_{n+1}-y_{n}\right\|+M_{3} \prod_{i=1}^{n} \mu_{i} \\
& +M_{6}\left(\left|\lambda_{n}-\lambda_{n+1}\right|+\left|\alpha_{n+1}-\alpha_{n}\right|\right),
\end{aligned}
$$

where $M_{6}$ is an appropriate constant such that $M_{6}=\max \left\{\sup _{n \geq 1}\left\|B y_{n}\right\|, \sup _{n \geq 1}\left\|W_{n} \phi_{n}-x_{n}\right\|\right\}$.

Substituting (3.26) and (3.36) into (3.40), we obtain

$$
\begin{aligned}
\left\|k_{n}-k_{n+1}\right\| \leq & \alpha_{n}\left\|x_{n}-x_{n+1}\right\|+\left(1-\alpha_{n}\right)\left\{\left\|u_{n}-u_{n+1}\right\|+M_{5}\left(\left|\delta_{n}-\delta_{n+1}\right|+\left|\varphi_{n+1}-\varphi_{n}\right|\right)+M_{2} \prod_{i=1}^{n} \mu_{i}\right\} \\
& +M_{3} \prod_{i=1}^{n} \mu_{i}+M_{6}\left(\left|\lambda_{n}-\lambda_{n+1}\right|+\left|\alpha_{n+1}-\alpha_{n}\right|\right) \\
= & \alpha_{n}\left\|x_{n}-x_{n+1}\right\|+\left(1-\alpha_{n}\right)\left\|u_{n}-u_{n+1}\right\|+\left(1-\alpha_{n}\right) M_{5}\left(\left|\delta_{n}-\delta_{n+1}\right|+\left|\varphi_{n+1}-\varphi_{n}\right|\right) \\
& +\left(1-\alpha_{n}\right) M_{2} \prod_{i=1}^{n} \mu_{i}+M_{3} \prod_{i=1}^{n} \mu_{i}+M_{6}\left(\left|\lambda_{n}-\lambda_{n+1}\right|+\left|\alpha_{n+1}-\alpha_{n}\right|\right) \\
\leq & \alpha_{n}\left\|x_{n}-x_{n+1}\right\|+\left(1-\alpha_{n}\right)\left\{\left\|x_{n+1}-x_{n}\right\|+M_{1}\left|r_{n+1}-r_{n}\right|\right\} \\
& +\left(1-\alpha_{n}\right) M_{5}\left(\left|\delta_{n}-\delta_{n+1}\right|+\left|\varphi_{n+1}-\varphi_{n}\right|\right)+\left(1-\alpha_{n}\right) M_{2} \prod_{i=1}^{n} \mu_{i} \\
& +M_{3} \prod_{i=1}^{n} \mu_{i}+M_{6}\left(\left|\lambda_{n}-\lambda_{n+1}\right|+\left|\alpha_{n+1}-\alpha_{n}\right|\right) \\
= & \alpha_{n}\left\|x_{n}-x_{n+1}\right\|+\left(1-\alpha_{n}\right)\left\|x_{n+1}-x_{n}\right\|+\left(1-\alpha_{n}\right) M_{1}\left|r_{n+1}-r_{n}\right| \\
& +\left(1-\alpha_{n}\right) M_{5}\left(\left|\delta_{n}-\delta_{n+1}\right|+\left|\varphi_{n+1}-\varphi_{n}\right|\right)+\left(1-\alpha_{n}\right) M_{2} \prod_{i=1}^{n} \mu_{i} \\
& +M_{3} \prod_{i=1}^{n} \mu_{i}+M_{6}\left(\left|\lambda_{n}-\lambda_{n+1}\right|+\left|\alpha_{n+1}-\alpha_{n}\right|\right) \\
\leq & \left\|x_{n}-x_{n+1}\right\|+M_{1}\left|r_{n+1}-r_{n}\right|+M_{2} \prod_{i=1}^{n} \mu_{i}+M_{3} \prod_{i=1}^{n} \mu_{i} \\
& +M_{5}\left(\left|\delta_{n}-\delta_{n+1}\right|+\left|\varphi_{n+1}-\varphi_{n}\right|\right)+M_{6}\left(\left|\lambda_{n}-\lambda_{n+1}\right|+\left|\alpha_{n+1}-\alpha_{n}\right|\right) \\
\leq & \left\|x_{n}-x_{n+1}\right\|+M_{2} \prod_{i=1}^{n} \mu_{i}+M_{3} \prod_{i=1}^{n} \mu_{i} \\
& +K_{1}\left(\left|r_{n+1}-r_{n}\right|+\left|\delta_{n}-\delta_{n+1}\right|+\left|\varphi_{n+1}-\varphi_{n}\right|+\left|\lambda_{n}-\lambda_{n+1}\right|+\left|\alpha_{n+1}-\alpha_{n}\right|\right),
\end{aligned}
$$

where $K_{1}$ is an appropriate constant such that $K_{1}=\max \left\{M_{1}, M_{5}, M_{6}\right\}$. 
Substituting (3.41) into (3.29), we obtain

$$
\begin{aligned}
\left\|\theta_{n+1}-\theta_{n}\right\| \leq & \left\|k_{n+1}-k_{n}\right\|+\left|\tau_{n}-\tau_{n+1}\right| \mid B k_{n} \| \\
\leq & \left\|x_{n}-x_{n+1}\right\|+M_{2} \prod_{i=1}^{n} \mu_{i}+M_{3} \prod_{i=1}^{n} \mu_{i} \\
& +K_{1}\left(\left|r_{n+1}-r_{n}\right|+\left|\delta_{n}-\delta_{n+1}\right|+\left|\varphi_{n+1}-\varphi_{n}\right|+\left|\lambda_{n}-\lambda_{n+1}\right|+\left|\alpha_{n+1}-\alpha_{n}\right|\right) \\
& +\left|\tau_{n}-\tau_{n+1}\right||| B k_{n} \| \\
\leq & || x_{n}-x_{n+1} \|+\mathrm{M}_{2} \prod_{i=1}^{n} \mu_{i}+M_{3} \prod_{i=1}^{n} \mu_{i} \\
& +K_{2}\left(\left|r_{n+1}-r_{n}\right|+\left|\delta_{n}-\delta_{n+1}\right|+\left|\varphi_{n+1}-\varphi_{n}\right|+\left|\lambda_{n}-\lambda_{n+1}\right|+\left|\alpha_{n+1}-\alpha_{n}\right|+\left|\tau_{n}-\tau_{n+1}\right|\right),
\end{aligned}
$$

where $K_{2}$ is an appropriate constant such that $K_{2}=\max \left\{\sup _{n \geq 1}\left\|B k_{n}\right\|, K_{1}\right\}$.

Define

$$
x_{n+1}=\left(1-\beta_{n}\right) z_{n}+\beta_{n} x_{n}, \quad n \geq 1 .
$$

Observe that from the definition $z_{n}$, we obtain

$$
\begin{aligned}
z_{n+1}-z_{n}= & \frac{\epsilon_{n+1} \gamma f\left(W_{n+1} x_{n+1}\right)+\left(\left(1-\beta_{n+1}\right) I-\epsilon_{n+1} A\right) W_{n+1} \theta_{n+1}}{1-\beta_{n+1}} \\
& -\frac{\epsilon_{n} \gamma f\left(W_{n} x_{n}\right)+\left(\left(1-\beta_{n}\right) I-\epsilon_{n} A\right) W_{n} \theta_{n}}{1-\beta_{n}} \\
= & \frac{\epsilon_{n+1}}{1-\beta_{n+1}} \gamma f\left(W_{n+1} x_{n+1}\right)-\frac{\epsilon_{n}}{1-\beta_{n}} \gamma f\left(W_{n} x_{n}\right)+W_{n+1} \theta_{n+1}-W_{n} \theta_{n} \\
& +\frac{\epsilon_{n}}{1-\beta_{n}} A W_{n} \theta_{n}-\frac{\epsilon_{n+1}}{1-\beta_{n+1}} A W_{n+1} \theta_{n+1} \\
= & \frac{\epsilon_{n+1}}{1-\beta_{n+1}}\left(\gamma f\left(W_{n+1} x_{n+1}\right)-A W_{n+1} \theta_{n+1}\right)+\frac{\epsilon_{n}}{1-\beta_{n}}\left(A W_{n} \theta_{n}-\gamma f\left(W_{n} x_{n}\right)\right) \\
& +W_{n+1} \theta_{n+1}-W_{n+1} \theta_{n}+W_{n+1} \theta_{n}-W_{n} \theta_{n} .
\end{aligned}
$$


It follows from (3.32), (3.42), and (3.44) that

$$
\begin{aligned}
\| z_{n+1}- & z_{n}\|-\| x_{n+1}-x_{n} \| \\
\leq & \frac{\epsilon_{n+1}}{1-\beta_{n+1}}\left(\left\|\gamma f\left(W_{n+1} x_{n+1}\right)\right\|+\left\|A W_{n+1} \theta_{n+1}\right\|\right)+\frac{\epsilon_{n}}{1-\beta_{n}}\left(\left\|A W_{n} \theta_{n}\right\|+\left\|\gamma f\left(W_{n} x_{n}\right)\right\|\right) \\
& +\left\|W_{n+1} \theta_{n+1}-W_{n+1} \theta_{n}\right\|+\left\|W_{n+1} \theta_{n}-W_{n} \theta_{n}\right\|-\left\|x_{n+1}-x_{n}\right\| \\
\leq & \frac{\epsilon_{n+1}}{1-\beta_{n+1}}\left(\left\|\gamma f\left(W_{n+1} x_{n+1}\right)\right\|+\left\|A W_{n+1} \theta_{n+1}\right\|\right)+\frac{\epsilon_{n}}{1-\beta_{n}}\left(\left\|A W_{n} \theta_{n}\right\|+\left\|\gamma f\left(W_{n} x_{n}\right)\right\|\right) \\
& +\left\|\theta_{n+1}-\theta_{n}\right\|+\left\|W_{n+1} \theta_{n}-W_{n} \theta_{n}\right\|-\left\|x_{n+1}-x_{n}\right\| \\
\leq & \frac{\epsilon_{n+1}}{1-\beta_{n+1}}\left(\left\|\gamma f\left(W_{n+1} x_{n+1}\right)\right\|+\left\|A W_{n+1} \theta_{n+1}\right\|\right)+\frac{\epsilon_{n}}{1-\beta_{n}}\left(\left\|A W_{n} \theta_{n}\right\|+\left\|\gamma f\left(W_{n} x_{n}\right)\right\|\right) \\
& +M_{2} \prod_{i=1}^{n} \mu_{i}+M_{3} \prod_{i=1}^{n} \mu_{i}+M_{4} \prod_{i=1}^{n} \mu_{i} \\
& +K_{2}\left(\left|r_{n+1}-r_{n}\right|+\left|\delta_{n}-\delta_{n+1}\right|+\left|\varphi_{n+1}-\varphi_{n}\right|+\left|\lambda_{n}-\lambda_{n+1}\right|+\left|\alpha_{n+1}-\alpha_{n}\right|+\left|\tau_{n}-\tau_{n+1}\right|\right) \\
\leq & \frac{\epsilon_{n+1}}{1-\beta_{n+1}}\left(\left\|\gamma f\left(W_{n+1} x_{n+1}\right)\right\|+\left\|A W_{n+1} \theta_{n+1}\right\|\right)+\frac{\epsilon_{n}}{1-\beta_{n}}\left(\left\|A W_{n} \theta_{n}\right\|+\left\|\gamma f\left(W_{n} x_{n}\right)\right\|\right) \\
& +3 K \prod_{i=1}^{n} \mu_{i} \\
& +K_{2}\left(\left|r_{n+1}-r_{n}\right|+\left|\delta_{n}-\delta_{n+1}\right|+\left|\varphi_{n+1}-\varphi_{n}\right|+\left|\lambda_{n}-\lambda_{n+1}\right|+\left|\alpha_{n+1}-\alpha_{n}\right|+\left|\tau_{n}-\tau_{n+1}\right|\right)
\end{aligned}
$$

where $K$ is an appropriate constant such that $K=\max \left\{M_{2}, M_{3}, M_{4}\right\}$.

It follows from conditions (C1), (C2), (C3), (C4), (C5), and $0<\mu_{i} \leq b<1$, for all $i \geq 1$

$$
\limsup _{n \rightarrow \infty}\left(\left\|z_{n+1}-z_{n}\right\|-\left\|x_{n+1}-x_{n}\right\|\right) \leq 0 .
$$

Hence, by Lemma 2.11, we obtain

$$
\lim _{n \rightarrow \infty}\left\|z_{n}-x_{n}\right\|=0
$$

It follows that

$$
\lim _{n \rightarrow \infty}\left\|x_{n+1}-x_{n}\right\|=\lim _{n \rightarrow \infty}\left(1-\beta_{n}\right)\left\|z_{n}-x_{n}\right\|=0 .
$$


Applying (3.48) and conditions in Theorem 3.3 to (3.26), (3.41), and (3.42), we obtain that

$$
\lim _{n \rightarrow \infty}\left\|u_{n+1}-u_{n}\right\|=\lim _{n \rightarrow \infty}\left\|k_{n+1}-k_{n}\right\|=\lim _{n \rightarrow \infty}\left\|\theta_{n+1}-\theta_{n}\right\|=0 .
$$

From (3.49), (C2), (C5), and $0<\mu_{i} \leq b<1$, for all $i \geq 1$, we also have

$$
\lim _{n \rightarrow \infty}\left\|y_{n+1}-y_{n}\right\|=0
$$

Since $x_{n+1}=\epsilon_{n} \gamma f\left(W_{n} x_{n}\right)+\beta_{n} x_{n}+\left(\left(1-\beta_{n}\right) I-\epsilon_{n} A\right) W_{n} \theta_{n}$, we have

$$
\begin{aligned}
\left\|x_{n}-W_{n} \theta_{n}\right\| & \leq\left\|x_{n}-x_{n+1}\right\|+\left\|x_{n+1}-W_{n} \theta_{n}\right\| \\
& =\left\|x_{n}-x_{n+1}\right\|+\left\|\epsilon_{n} \gamma f\left(W_{n} x_{n}\right)+\beta_{n} x_{n}+\left(\left(1-\beta_{n}\right) I-\epsilon_{n} A\right) W_{n} \theta_{n}-W_{n} \theta_{n}\right\| \\
& =\left\|x_{n}-x_{n+1}\right\|+\left\|\epsilon_{n}\left(\gamma f\left(W_{n} x_{n}\right)-A W_{n} \theta_{n}\right)+\beta_{n}\left(x_{n}-W_{n} \theta_{n}\right)\right\| \\
& \leq\left\|x_{n}-x_{n+1}\right\|+\epsilon_{n}\left(\left\|\gamma f\left(W_{n} x_{n}\right)\right\|+\left\|A W_{n} \theta_{n}\right\|\right)+\beta_{n}\left\|x_{n}-W_{n} \theta_{n}\right\|,
\end{aligned}
$$

that is,

$$
\left\|x_{n}-W_{n} \theta_{n}\right\| \leq \frac{1}{1-\beta_{n}}\left\|x_{n}-x_{n+1}\right\|+\frac{\epsilon_{n}}{1-\beta_{n}}\left(\left\|\gamma f\left(W_{n} x_{n}\right)\right\|+\left\|A W_{n} \theta_{n}\right\|\right) .
$$

By (C1), (C3), and (3.48) it follows that

$$
\lim _{n \rightarrow \infty}\left\|W_{n} \theta_{n}-x_{n}\right\|=0
$$

Step 4. We claim that the following statements hold:

(i) $\lim _{n \rightarrow \infty}\left\|u_{n}-\theta_{n}\right\|=0$;

(ii) $\lim _{n \rightarrow \infty}\left\|x_{n}-u_{n}\right\|=0$;

(iii) $\lim _{n \rightarrow \infty}\left\|W_{n} \theta_{n}-\theta_{n}\right\|=0$.

Since $B$ is relaxed $(u, v)$-cocoercive and $\xi$-Lipschitz continuous mappings, by the assumptions imposed on $\left\{\tau_{n}\right\}$ for any $p \in \Theta:=\bigcap_{n=1}^{\infty} F\left(T_{n}\right) \cap \operatorname{EP}(F, D) \cap \operatorname{VI}(E, B)$, we have 
Journal of Inequalities and Applications

$$
\begin{aligned}
\left\|W_{n} \theta_{n}-p\right\|^{2} & \leq\left\|P_{E}\left(k_{n}-\tau_{n} B k_{n}\right)-P_{E}\left(p-\tau_{n} B p\right)\right\|^{2} \\
& \leq\left\|\left(k_{n}-\tau_{n} B k_{n}\right)-\left(p-\tau_{n} B p\right)\right\|^{2} \\
& =\left\|\left(k_{n}-p\right)-\tau_{n}\left(B k_{n}-B p\right)\right\|^{2} \\
& \leq\left\|k_{n}-p\right\|^{2}-2 \tau_{n}\left\langle k_{n}-p, B k_{n}-B p\right\rangle+\tau_{n}^{2}\left\|B k_{n}-B p\right\|^{2} \\
& \leq\left\|x_{n}-p\right\|^{2}-2 \tau_{n}\left\langle k_{n}-p, B k_{n}-B p\right\rangle+\tau_{n}^{2}\left\|B k_{n}-B p\right\|^{2} \\
& \leq\left\|x_{n}-p\right\|^{2}-2 \tau_{n}\left\{-u\left\|B k_{n}-B p\right\|^{2}+v\left\|k_{n}-p\right\|^{2}\right\}+\tau_{n}^{2}\left\|B k_{n}-B p\right\|^{2} \\
& \leq\left\|x_{n}-p\right\|^{2}+2 \tau_{n} u\left\|B k_{n}-B p\right\|^{2}-2 \tau_{n} v\left\|k_{n}-p\right\|^{2}+\tau_{n}^{2}\left\|B k_{n}-B p\right\|^{2} \\
& \leq\left\|x_{n}-p\right\|^{2}+2 \tau_{n} u\left\|B k_{n}-B p\right\|^{2}-\frac{2 \tau_{n} v}{\xi^{2}}\left\|B k_{n}-B p\right\|^{2}+\tau_{n}^{2}\left\|B k_{n}-B p\right\|^{2} \\
& =\left\|x_{n}-p\right\|^{2}+\left(2 \tau_{n} u+\tau_{n}^{2}-\frac{2 \tau_{n} v}{\xi^{2}}\right)\left\|B k_{n}-B p\right\|^{2} .
\end{aligned}
$$

Similarly, we have

$$
\begin{aligned}
& \left\|W_{n} \phi_{n}-p\right\|^{2} \leq\left\|x_{n}-p\right\|^{2}+\left(2 \lambda_{n} u+\lambda_{n}^{2}-\frac{2 \lambda_{n} v}{\xi^{2}}\right)\left\|B y_{n}-B p\right\|^{2}, \\
& \left\|W_{n} \psi_{n}-p\right\|^{2} \leq\left\|x_{n}-p\right\|^{2}+\left(2 \delta_{n} u+\delta_{n}^{2}-\frac{2 \delta_{n} v}{\xi^{2}}\right)\left\|B u_{n}-B p\right\|^{2} .
\end{aligned}
$$

Observe that

$$
\begin{aligned}
\left\|x_{n+1}-p\right\|^{2}= & \left\|\left(\left(1-\beta_{n}\right) I-\epsilon_{n} A\right)\left(W_{n} \theta_{n}-p\right)+\beta_{n}\left(x_{n}-p\right)+\epsilon_{n}\left(\gamma f\left(W_{n} x_{n}\right)-A p\right)\right\|^{2} \\
= & \left\|\left(\left(1-\beta_{n}\right) I-\epsilon_{n} A\right)\left(W_{n} \theta_{n}-p\right)+\beta_{n}\left(x_{n}-p\right)\right\|^{2}+\epsilon_{n}^{2}\left\|\gamma f\left(W_{n} x_{n}\right)-A p\right\|^{2} \\
& +2 \beta_{n} \epsilon_{n}\left\langle x_{n}-p, \gamma f\left(W_{n} x_{n}\right)-A p\right\rangle \\
& +2 \epsilon_{n}\left\langle\left(\left(1-\beta_{n}\right) I-\epsilon_{n} A\right)\left(W_{n} \theta_{n}-p\right), \gamma f\left(W_{n} x_{n}\right)-A p\right\rangle \\
\leq & \left(\left(1-\beta_{n}-\epsilon_{n} \bar{\gamma}\right)\left\|W_{n} \theta_{n}-p\right\|+\beta_{n}\left\|x_{n}-p\right\|\right)^{2}+\epsilon_{n}^{2}\left\|\gamma f\left(W_{n} x_{n}\right)-A p\right\|^{2} \\
& +2 \beta_{n} \epsilon_{n}\left\langle x_{n}-p, \gamma f\left(W_{n} x_{n}\right)-A p\right\rangle \\
& +2 \epsilon_{n}\left\langle\left(\left(1-\beta_{n}\right) I-\epsilon_{n} A\right)\left(W_{n} \theta_{n}-p\right), \gamma f\left(W_{n} x_{n}\right)-A p\right\rangle \\
\leq & \left(\left(1-\beta_{n}-\epsilon_{n} \bar{\gamma}\right)\left\|W_{n} \theta_{n}-p\right\|+\beta_{n}\left\|x_{n}-p\right\|^{2}+c_{n}\right. \\
\leq & \left(1-\beta_{n}-\epsilon_{n} \bar{\gamma}\right)^{2}\left\|W_{n} \theta_{n}-p\right\|^{2}+\beta_{n}^{2}\left\|x_{n}-p\right\|^{2} \\
& +2\left(1-\beta_{n}-\epsilon_{n} \bar{\gamma}\right) \beta_{n}\left\|W_{n} \theta_{n}-p\right\|\left\|x_{n}-p\right\|+c_{n} \\
\leq & \left(1-\beta_{n}-\epsilon_{n} \bar{\gamma}\right)^{2}\left\|W_{n} \theta_{n}-p\right\|^{2}+\beta_{n}^{2}\left\|x_{n}-p\right\|^{2} \\
& +\left(1-\beta_{n}-\epsilon_{n} \bar{\gamma}\right) \beta_{n}\left(\left\|W_{n} \theta_{n}-p\right\|^{2}+\left\|x_{n}-p\right\|^{2}\right)+c_{n}
\end{aligned}
$$




$$
\begin{aligned}
= & {\left[\left(1-\epsilon_{n} \bar{\gamma}\right)^{2}-2\left(1-\epsilon_{n} \bar{\gamma}\right) \beta_{n}+\beta_{n}^{2}\right]\left\|W_{n} \theta_{n}-p\right\|^{2}+\beta_{n}^{2}\left\|x_{n}-p\right\|^{2} } \\
& +\left(\left(1-\epsilon_{n} \bar{\gamma}\right) \beta_{n}-\beta_{n}^{2}\right)\left(\left\|W_{n} \theta_{n}-p\right\|^{2}+\left\|x_{n}-p\right\|^{2}\right)+c_{n} \\
= & {\left[\left(1-\epsilon_{n} \bar{\gamma}\right)^{2}-\left(1-\epsilon_{n} \bar{\gamma}\right) \beta_{n}\right]\left\|W_{n} \theta_{n}-p\right\|^{2}+\left(1-\epsilon_{n} \bar{\gamma}\right) \beta_{n}\left\|x_{n}-p\right\|^{2}+c_{n} } \\
= & \left(1-\epsilon_{n} \bar{\gamma}\right)\left(1-\beta_{n}-\epsilon_{n} \bar{\gamma}\right)\left\|W_{n} \theta_{n}-p\right\|^{2}+\left(1-\epsilon_{n} \bar{\gamma}\right) \beta_{n}\left\|x_{n}-p\right\|^{2}+c_{n},
\end{aligned}
$$

where

$$
\begin{aligned}
c_{n}= & \epsilon_{n}^{2}\left\|\gamma f\left(x_{n}\right)-A p\right\|^{2}+2 \beta_{n} \epsilon_{n}\left\langle x_{n}-p, \gamma f\left(x_{n}\right)-A p\right\rangle \\
& +2 \epsilon_{n}\left\langle\left(\left(1-\beta_{n}\right) I-\epsilon_{n} A\right)\left(W_{n} \theta_{n}-p\right), \gamma f\left(x_{n}\right)-A p\right\rangle .
\end{aligned}
$$

It follows from condition (C1) that

$$
\lim _{n \rightarrow \infty} c_{n}=0
$$

Substituting (3.54) into (3.56), and using condition (C6), we have

$$
\begin{aligned}
\left\|x_{n+1}-p\right\|^{2} \leq & \left(1-\epsilon_{n} \bar{\gamma}\right)\left(1-\beta_{n}-\epsilon_{n} \bar{\gamma}\right)\left\{\left\|x_{n}-p\right\|^{2}+\left(2 \tau_{n} u+\tau_{n}^{2}-\frac{2 \tau_{n} v}{\xi^{2}}\right)\left\|B k_{n}-B p\right\|^{2}\right\} \\
& +\left(1-\epsilon_{n} \bar{\gamma}\right) \beta_{n}\left\|x_{n}-p\right\|^{2}+c_{n} \\
= & \left(1-\epsilon_{n} \bar{\gamma}\right)^{2}\left\|x_{n}-p\right\|^{2}+\left(1-\epsilon_{n} \bar{\gamma}\right)\left(1-\beta_{n}-\epsilon_{n} \bar{\gamma}\right) \\
& \times\left(2 \tau_{n} u+\tau_{n}^{2}-\frac{2 \tau_{n} v}{\xi^{2}}\right)\left\|B k_{n}-B p\right\|^{2}+c_{n} \\
\leq & \left\|x_{n}-p\right\|^{2}+\left(2 \tau_{n} u+\tau_{n}^{2}-\frac{2 \tau_{n} v}{\xi^{2}}\right)\left\|B k_{n}-B p\right\|^{2}+c_{n} .
\end{aligned}
$$

It follows that

$$
\begin{aligned}
\left(\frac{2 a v}{\xi^{2}}-2 b u-b^{2}\right)\left\|B k_{n}-B p\right\|^{2} & \leq\left(\frac{2 \tau_{n} v}{\xi^{2}}-2 \tau_{n} u-\tau_{n}^{2}\right)\left\|B k_{n}-B p\right\|^{2} \\
& \leq\left\|x_{n}-p\right\|^{2}-\left\|x_{n+1}-p\right\|^{2}+c_{n} \\
& =\left(\left\|x_{n}-p\right\|-\left\|x_{n+1}-p\right\|\right)\left(\left\|x_{n}-p\right\|+\left\|x_{n+1}-p\right\|\right)+c_{n} \\
& \leq\left\|x_{n}-x_{n+1}\right\|\left(\left\|x_{n}-p\right\|+\left\|x_{n+1}-p\right\|\right)+c_{n} .
\end{aligned}
$$


Journal of Inequalities and Applications

Since $c_{n} \rightarrow 0$ as $n \rightarrow \infty$ and (3.48), we obtain

$$
\lim _{n \rightarrow \infty}\left\|B k_{n}-B p\right\|=0
$$

Note that

$$
\begin{aligned}
\left\|k_{n}-p\right\|^{2} & \leq \alpha_{n}\left\|x_{n}-p\right\|+\left(1-\alpha_{n}\right)\left\|W_{n} \phi_{n}-p\right\|^{2} \\
& \leq \alpha_{n}\left\|x_{n}-p\right\|^{2}+\left(1-\alpha_{n}\right)\left\{\left\|x_{n}-p\right\|^{2}+\left(2 \lambda_{n} u+\lambda_{n}^{2}-\frac{2 \lambda_{n} v}{\xi^{2}}\right)\left\|B y_{n}-B p\right\|^{2}\right\} \\
& \leq\left\|x_{n}-p\right\|^{2}+\left(1-\alpha_{n}\right)\left(2 \lambda_{n} u+\lambda_{n}^{2}-\frac{2 \lambda_{n} v}{\xi^{2}}\right)\left\|B y_{n}-B p\right\|^{2}, \\
\left\|y_{n}-p\right\|^{2} & \leq \varphi_{n}\left\|u_{n}-p\right\|+\left(1-\varphi_{n}\right)\left\|W_{n} \psi_{n}-p\right\|^{2} \\
& \leq \varphi_{n}\left\|x_{n}-p\right\|^{2}+\left(1-\varphi_{n}\right)\left\{\left\|x_{n}-p\right\|^{2}+\left(2 \delta_{n} u+\delta_{n}^{2}-\frac{2 \delta_{n} v}{\xi^{2}}\right)\left\|B u_{n}-B p\right\|^{2}\right\} \\
& \leq\left\|x_{n}-p\right\|^{2}+\left(1-\varphi_{n}\right)\left(2 \delta_{n} u+\delta_{n}^{2}-\frac{2 \delta_{n} v}{\xi^{2}}\right)\left\|B u_{n}-B p\right\|^{2} .
\end{aligned}
$$

Using (3.56) again, we have

$$
\begin{aligned}
\left\|x_{n+1}-p\right\|^{2} & \leq\left(1-\epsilon_{n} \bar{\gamma}\right)\left(1-\beta_{n}-\epsilon_{n} \bar{\gamma}\right)\left\|W_{n} \theta_{n}-p\right\|^{2}+\left(1-\epsilon_{n} \bar{\gamma}\right) \beta_{n}\left\|x_{n}-p\right\|^{2}+c_{n} \\
& \leq\left(1-\epsilon_{n} \bar{\gamma}\right)\left(1-\beta_{n}-\epsilon_{n} \bar{\gamma}\right)\left\|\theta_{n}-p\right\|^{2}+\left(1-\epsilon_{n} \bar{\gamma}\right) \beta_{n}\left\|x_{n}-p\right\|^{2}+c_{n} \\
& \leq\left(1-\epsilon_{n} \bar{\gamma}\right)\left(1-\beta_{n}-\epsilon_{n} \bar{\gamma}\right)\left\|k_{n}-p\right\|^{2}+\left(1-\epsilon_{n} \bar{\gamma}\right) \beta_{n}\left\|x_{n}-p\right\|^{2}+c_{n} .
\end{aligned}
$$

Substituting (3.62) into (3.64) and using condition (C2) and (C6), we have

$$
\begin{aligned}
\left\|x_{n+1}-p\right\|^{2} \leq & \left(1-\epsilon_{n} \bar{\gamma}\right)\left(1-\beta_{n}-\epsilon_{n} \bar{\gamma}\right)\left\{\left\|x_{n}-p\right\|^{2}+\left(1-\alpha_{n}\right)\left(2 \lambda_{n} u+\lambda_{n}^{2}-\frac{2 \lambda_{n} v}{\xi^{2}}\right)\left\|B y_{n}-B p\right\|^{2}\right\} \\
& +\left(1-\epsilon_{n} \bar{\gamma}\right) \beta_{n}\left\|x_{n}-p\right\|^{2}+c_{n} \\
= & \left(1-\epsilon_{n} \bar{\gamma}\right)\left(1-\beta_{n}-\epsilon_{n} \bar{\gamma}\right)\left(1-\alpha_{n}\right)\left(2 \lambda_{n} u+\lambda_{n}^{2}-\frac{2 \lambda_{n} v}{\xi^{2}}\right)\left\|B y_{n}-B p\right\|^{2} \\
& +\left(1-\epsilon_{n} \bar{\gamma}\right)^{2}\left\|x_{n}-p\right\|^{2}+c_{n} \\
\leq & \left\|x_{n}-p\right\|^{2}+\left(1-\alpha_{n}\right)\left(2 \lambda_{n} u+\lambda_{n}^{2}-\frac{2 \lambda_{n} v}{\xi^{2}}\right)\left\|B y_{n}-B p\right\|^{2}+c_{n} .
\end{aligned}
$$


It follows that

$$
\begin{aligned}
\left(1-\alpha_{n}\right)\left(\frac{2 a v}{\xi^{2}}-2 b u-b^{2}\right)\left\|B y_{n}-B p\right\|^{2} & \leq\left(1-\alpha_{n}\right)\left(\frac{2 \tau_{n} v}{\xi^{2}}-2 \tau_{n} u-\tau_{n}^{2}\right)\left\|B y_{n}-B p\right\|^{2} \\
& \leq\left\|x_{n}-p\right\|^{2}-\left\|x_{n+1}-p\right\|^{2}+c_{n} \\
& \leq\left\|x_{n}-x_{n+1}\right\|\left(\left\|x_{n}-p\right\|+\left\|x_{n+1}-p\right\|\right)+c_{n} .
\end{aligned}
$$

Since $c_{n} \rightarrow 0$ as $n \rightarrow \infty$ and (3.48), we obtain

$$
\lim _{n \rightarrow \infty}\left\|B y_{n}-B p\right\|=0
$$

In a similar way, we can prove

$$
\lim _{n \rightarrow \infty}\left\|B u_{n}-B p\right\|=0
$$

By (2.3), we also have

$$
\begin{aligned}
\left\|\theta_{n}-p\right\|^{2} & =\left\|P_{E}\left(k_{n}-\tau_{n} B k_{n}\right)-P_{E}\left(p-\tau_{n} B p\right)\right\|^{2} \\
& =\left\|P_{E}\left(I-\tau_{n} B\right) k_{n}-P_{E}\left(I-\tau_{n} B\right) p\right\|^{2} \\
& \leq\left\langle\left(I-\tau_{n} B\right) k_{n}-\left(I-\tau_{n} B\right) p, \theta_{n}-p\right\rangle \\
& =\frac{1}{2}\left\{\left\|\left(I-\tau_{n} B\right) k_{n}-\left(I-\tau_{n} B\right) p\right\|^{2}+\left\|\theta_{n}-p\right\|^{2}\right. \\
& \left.\quad-\left\|\left(I-\tau_{n} B\right) k_{n}-\left(I-\tau_{n} B\right) p-\left(\theta_{n}-p\right)\right\|^{2}\right\} \\
\leq & \frac{1}{2}\left\|k_{n}-p\right\|^{2}+\left\|\theta_{n}-p\right\|^{2}-\left\|\left(k_{n}-\theta_{n}\right)-\tau_{n}\left(B k_{n}-B p\right)\right\|^{2} \\
\leq & \frac{1}{2}\left\{\left\|x_{n}-p\right\|^{2}+\left\|\theta_{n}-p\right\|^{2}-\left\|k_{n}-\theta_{n}\right\|^{2}-\tau_{n}^{2}\left\|B k_{n}-B p\right\|^{2}+2 \tau_{n}\left\langle k_{n}-\theta_{n}, B k_{n}-B p\right\rangle\right\}
\end{aligned}
$$

which yields that

$$
\left\|\theta_{n}-p\right\|^{2} \leq\left\|x_{n}-p\right\|^{2}-\left\|k_{n}-\theta_{n}\right\|^{2}+2 \tau_{n}\left\|k_{n}-\theta_{n}\right\|\left\|B k_{n}-B p\right\| .
$$


Substituting (3.70) into (3.56), we have

$$
\begin{aligned}
\left\|x_{n+1}-p\right\|^{2} \leq & \left(1-\epsilon_{n} \bar{\gamma}\right)\left(1-\beta_{n}-\epsilon_{n} \bar{\gamma}\right)\left\|W_{n} \theta_{n}-p\right\|^{2}+\left(1-\epsilon_{n} \bar{\gamma}\right) \beta_{n}\left\|x_{n}-p\right\|^{2}+c_{n} \\
\leq & \left(1-\epsilon_{n} \bar{\gamma}\right)\left(1-\beta_{n}-\epsilon_{n} \bar{\gamma}\right)\left\|\theta_{n}-p\right\|^{2}+\left(1-\epsilon_{n} \bar{\gamma}\right) \beta_{n}\left\|x_{n}-p\right\|^{2}+c_{n} \\
\leq & \left(1-\epsilon_{n} \bar{\gamma}\right)\left(1-\beta_{n}-\epsilon_{n} \bar{\gamma}\right)\left\{\left\|x_{n}-p\right\|^{2}-\left\|k_{n}-\theta_{n}\right\|^{2}+2 \tau_{n}\left\|k_{n}-\theta_{n}\right\|\left\|B k_{n}-B p\right\|\right\} \\
& +\left(1-\epsilon_{n} \bar{\gamma}\right) \beta_{n}\left\|x_{n}-p\right\|^{2}+c_{n} \\
= & \left(1-\epsilon_{n} \bar{\gamma}\right)^{2}\left\|x_{n}-p\right\|^{2}-\left(1-\epsilon_{n} \bar{\gamma}\right)\left(1-\beta_{n}-\epsilon_{n} \bar{\gamma}\right)\left\|k_{n}-\theta_{n}\right\|^{2} \\
& +2\left(1-\epsilon_{n} \bar{\gamma}\right)\left(1-\beta_{n}-\epsilon_{n} \bar{\gamma}\right) \tau_{n}\left\|k_{n}-\theta_{n}\right\|\left\|B k_{n}-B p\right\|+c_{n} \\
\leq & \left\|x_{n}-p\right\|^{2}-\left(1-\epsilon_{n} \bar{\gamma}\right)\left(1-\beta_{n}-\epsilon_{n} \bar{\gamma}\right)\left\|k_{n}-\theta_{n}\right\|^{2} \\
& +2\left(1-\epsilon_{n} \bar{\gamma}\right)\left(1-\beta_{n}-\epsilon_{n} \bar{\gamma}\right) \tau_{n}\left\|k_{n}-\theta_{n}\right\|\left\|B k_{n}-B p\right\|+c_{n} .
\end{aligned}
$$

It follows that

$$
\begin{aligned}
\left(1-\epsilon_{n} \bar{\gamma}\right)\left(1-\beta_{n}-\epsilon_{n} \bar{\gamma}\right)\left\|k_{n}-\theta_{n}\right\|^{2} \leq & \left\|x_{n}-p\right\|^{2}-\left\|x_{n+1}-p\right\|^{2} \\
& +2\left(1-\epsilon_{n} \bar{\gamma}\right)\left(1-\beta_{n}-\epsilon_{n} \bar{\gamma}\right) \tau_{n}\left\|k_{n}-\theta_{n}\right\|\left\|B k_{n}-B p\right\|+c_{n} \\
\leq & \left\|x_{n}-x_{n+1}\right\|\left(\left\|x_{n}-p\right\|+\left\|x_{n+1}-p\right\|\right) \\
& +2\left(1-\epsilon_{n} \bar{\gamma}\right)\left(1-\beta_{n}-\epsilon_{n} \bar{\gamma}\right) \tau_{n}\left\|k_{n}-\theta_{n}\right\|\left\|B k_{n}-B p\right\|+c_{n} .
\end{aligned}
$$

Applying $\left\|x_{n+1}-x_{n}\right\| \rightarrow 0,\left\|B k_{n}-B p\right\| \rightarrow 0$ and $c_{n} \rightarrow 0$ as $n \rightarrow \infty$ to the last inequality, we have

$$
\lim _{n \rightarrow \infty}\left\|k_{n}-\theta_{n}\right\|=0
$$

On the other hand, we have

$$
\begin{aligned}
\left\|W_{n} \theta_{n}-p\right\|^{2} & \leq\left\|P_{E}\left(k_{n}-\tau_{n} B k_{n}\right)-P_{E}\left(p-\tau_{n} B p\right)\right\|^{2} \\
& =\left\|P_{E}\left(I-\tau_{n} B\right) k_{n}-P_{E}\left(I-\tau_{n} B\right) p\right\|^{2} \\
& \leq\left\langle\left(I-\tau_{n} B\right) k_{n}-\left(I-\tau_{n} B\right) p, W_{n} \theta_{n}-p\right\rangle \\
& =\frac{1}{2}\left\{\left\|\left(I-\tau_{n} B\right) k_{n}-\left(I-\tau_{n} B\right) p\right\|^{2}+\left\|W_{n} \theta_{n}-p\right\|^{2}\right. \\
& \left.\quad-\left\|\left(I-\tau_{n} B\right) k_{n}-\left(I-\tau_{n} B\right) p-\left(W_{n} \theta_{n}-p\right)\right\|^{2}\right\}
\end{aligned}
$$




$$
\begin{gathered}
\leq \frac{1}{2}\left\|k_{n}-p\right\|^{2}+\left\|W_{n} \theta_{n}-p\right\|^{2}-\left\|\left(k_{n}-W_{n} \theta_{n}\right)-\tau_{n}\left(B k_{n}-B p\right)\right\|^{2} \\
\leq \frac{1}{2}\left\{\left\|x_{n}-p\right\|^{2}+\left\|W_{n} \theta_{n}-p\right\|^{2}-\left\|k_{n}-W_{n} \theta_{n}\right\|^{2}\right. \\
\left.\quad-\tau_{n}^{2}\left\|B k_{n}-B p\right\|^{2}+2 \tau_{n}\left\langle k_{n}-W_{n} \theta_{n}, B k_{n}-B p\right\rangle\right\},
\end{gathered}
$$

which yields that

$$
\left\|W_{n} \theta_{n}-p\right\|^{2} \leq\left\|x_{n}-p\right\|^{2}-\left\|k_{n}-W_{n} \theta_{n}\right\|^{2}+2 \tau_{n}\left\|k_{n}-W_{n} \theta_{n}\right\|\left\|B k_{n}-B p\right\| .
$$

Similarly, we can prove

$$
\begin{aligned}
& \left\|W_{n} \phi_{n}-p\right\|^{2} \leq\left\|x_{n}-p\right\|^{2}-\left\|y_{n}-W_{n} \phi_{n}\right\|^{2}+2 \lambda_{n}\left\|y_{n}-W_{n} \phi_{n}\right\|\left\|B y_{n}-B p\right\|, \\
& \left\|W_{n} \psi_{n}-p\right\|^{2} \leq\left\|x_{n}-p\right\|^{2}-\left\|u_{n}-W_{n} \psi_{n}\right\|^{2}+2 \delta_{n}\left\|u_{n}-W_{n} \psi_{n}\right\|\left\|B u_{n}-B p\right\| .
\end{aligned}
$$

Substituting (3.75) into (3.56), we have

$$
\begin{aligned}
\left\|x_{n+1}-p\right\|^{2} \leq & \left(1-\epsilon_{n} \bar{\gamma}\right)\left(1-\beta_{n}-\epsilon_{n} \bar{\gamma}\right)\left\|W_{n} \theta_{n}-p\right\|^{2}+\left(1-\epsilon_{n} \bar{\gamma}\right) \beta_{n}\left\|x_{n}-p\right\|^{2}+c_{n} \\
\leq & \left(1-\epsilon_{n} \bar{\gamma}\right)\left(1-\beta_{n}-\epsilon_{n} \bar{\gamma}\right) \\
& \times\left\{\left\|x_{n}-p\right\|^{2}-\left\|k_{n}-W_{n} \theta_{n}\right\|^{2}+2 \tau_{n}\left\|k_{n}-W_{n} \theta_{n}\right\|\left\|B k_{n}-B p\right\|\right\} \\
& +\left(1-\epsilon_{n} \bar{\gamma}\right) \beta_{n}\left\|x_{n}-p\right\|^{2}+c_{n} \\
= & \left(1-\epsilon_{n} \bar{\gamma}\right)^{2}\left\|x_{n}-p\right\|^{2}-\left(1-\epsilon_{n} \bar{\gamma}\right)\left(1-\beta_{n}-\epsilon_{n} \bar{\gamma}\right)\left\|k_{n}-W_{n} \theta_{n}\right\|^{2} \\
& +2\left(1-\epsilon_{n} \bar{\gamma}\right)\left(1-\beta_{n}-\epsilon_{n} \bar{\gamma}\right) \tau_{n}\left\|k_{n}-W_{n} \theta_{n}\right\|\left\|B k_{n}-B p\right\|+c_{n} \\
\leq & \left\|x_{n}-p\right\|^{2}-\left(1-\epsilon_{n} \bar{\gamma}\right)\left(1-\beta_{n}-\epsilon_{n} \bar{\gamma}\right)\left\|k_{n}-W_{n} \theta_{n}\right\|^{2} \\
& +2\left(1-\epsilon_{n} \bar{\gamma}\right)\left(1-\beta_{n}-\epsilon_{n} \bar{\gamma}\right) \tau_{n}\left\|k_{n}-W_{n} \theta_{n}\right\|\left\|B k_{n}-B p\right\|+c_{n},
\end{aligned}
$$

which yields that

$$
\begin{aligned}
&\left(1-\epsilon_{n} \bar{\gamma}\right)\left(1-\beta_{n}-\epsilon_{n} \bar{\gamma}\right)\left\|k_{n}-W_{n} \theta_{n}\right\|^{2} \\
& \leq \\
& \leq\left\|x_{n}-p\right\|^{2}-\left\|x_{n+1}-p\right\|^{2}+2\left(1-\epsilon_{n} \bar{\gamma}\right)\left(1-\beta_{n}-\epsilon_{n} \bar{\gamma}\right) \tau_{n}\left\|k_{n}-W_{n} \theta_{n}\right\|\left\|B k_{n}-B p\right\|+c_{n} \\
& \leq\left\|x_{n}-x_{n+1}\right\|\left(\left\|x_{n}-p\right\|+\left\|x_{n+1}-p\right\|\right) \\
&+2\left(1-\epsilon_{n} \bar{\gamma}\right)\left(1-\beta_{n}-\epsilon_{n} \bar{\gamma}\right) \tau_{n}\left\|k_{n}-W_{n} \theta_{n}\right\|\left\|B k_{n}-B p\right\|+c_{n} .
\end{aligned}
$$


Applying (3.48) and (3.61) to the last inequality, we have

$$
\lim _{n \rightarrow \infty}\left\|k_{n}-W_{n} \theta_{n}\right\|=0 .
$$

Using (3.64) again, we have

$$
\begin{aligned}
& \left\|x_{n+1}-p\right\|^{2} \leq\left(1-\epsilon_{n} \bar{\gamma}\right)\left(1-\beta_{n}-\epsilon_{n} \bar{\gamma}\right)\left\|k_{n}-p\right\|^{2}+\left(1-\epsilon_{n} \bar{\gamma}\right) \beta_{n}\left\|x_{n}-p\right\|^{2}+c_{n} \\
& =\left(1-\epsilon_{n} \bar{\gamma}\right)\left(1-\beta_{n}-\epsilon_{n} \bar{\gamma}\right)\left\{\left\|\alpha_{n}\left(x_{n}-p\right)+\left(1-\alpha_{n}\right)\left(W_{n} \phi_{n}-p\right)\right\|^{2}\right\} \\
& +\left(1-\epsilon_{n} \bar{\gamma}\right) \beta_{n}\left\|x_{n}-p\right\|^{2}+c_{n} \\
& \leq\left(1-\epsilon_{n} \bar{\gamma}\right)\left(1-\beta_{n}-\epsilon_{n} \bar{\gamma}\right)\left\{\alpha_{n}\left\|x_{n}-p\right\|^{2}+\left(1-\alpha_{n}\right)\left\|W_{n} \phi_{n}-p\right\|^{2}\right\} \\
& +\left(1-\epsilon_{n} \bar{\gamma}\right) \beta_{n}\left\|x_{n}-p\right\|^{2}+c_{n} \\
& =\left(1-\epsilon_{n} \bar{\gamma}\right)\left(1-\beta_{n}-\epsilon_{n} \bar{\gamma}\right) \alpha_{n}\left\|x_{n}-p\right\|^{2} \\
& +\left(1-\epsilon_{n} \bar{\gamma}\right)\left(1-\beta_{n}-\epsilon_{n} \bar{\gamma}\right)\left(1-\alpha_{n}\right)\left\|W_{n} \phi_{n}-p\right\|^{2}+\left(1-\epsilon_{n} \bar{\gamma}\right) \beta_{n}\left\|x_{n}-p\right\|^{2}+c_{n} \\
& \leq\left(1-\epsilon_{n} \bar{\gamma}\right)\left(1-\beta_{n}-\epsilon_{n} \bar{\gamma}\right) \alpha_{n}\left\|x_{n}-p\right\|^{2}+\left(1-\epsilon_{n} \bar{\gamma}\right)\left(1-\beta_{n}-\epsilon_{n} \bar{\gamma}\right)\left(1-\alpha_{n}\right) \\
& \times\left\{\left\|x_{n}-p\right\|^{2}-\left\|y_{n}-W_{n} \phi_{n}\right\|^{2}+2 \lambda_{n}\left\|y_{n}-W_{n} \phi_{n}\right\|\left\|B y_{n}-B p\right\|\right\} \\
& +\left(1-\epsilon_{n} \bar{\gamma}\right) \beta_{n}\left\|x_{n}-p\right\|^{2}+c_{n} \\
& =\left(1-\epsilon_{n} \bar{\gamma}\right)\left(1-\beta_{n}-\epsilon_{n} \bar{\gamma}\right) \alpha_{n}\left\|x_{n}-p\right\|^{2} \\
& +\left(1-\epsilon_{n} \bar{\gamma}\right)\left(1-\beta_{n}-\epsilon_{n} \bar{\gamma}\right)\left(1-\alpha_{n}\right)\left\|x_{n}-p\right\|^{2} \\
& -\left(1-\epsilon_{n} \bar{\gamma}\right)\left(1-\beta_{n}-\epsilon_{n} \bar{\gamma}\right)\left(1-\alpha_{n}\right)\left\|y_{n}-W_{n} \phi_{n}\right\|^{2} \\
& +\left(1-\epsilon_{n} \bar{\gamma}\right)\left(1-\beta_{n}-\epsilon_{n} \bar{\gamma}\right)\left(1-\alpha_{n}\right) 2 \lambda_{n}\left\|y_{n}-W_{n} \phi_{n}\right\|\left\|B y_{n}-B p\right\| \\
& +\left(1-\epsilon_{n} \bar{\gamma}\right) \beta_{n}\left\|x_{n}-p\right\|^{2}+c_{n} \\
& =\left(1-\epsilon_{n} \bar{\gamma}\right)\left(1-\beta_{n}-\epsilon_{n} \bar{\gamma}\right)\left\|x_{n}-p\right\|^{2} \\
& -\left(1-\epsilon_{n} \bar{\gamma}\right)\left(1-\beta_{n}-\epsilon_{n} \bar{\gamma}\right)\left(1-\alpha_{n}\right)\left\|y_{n}-W_{n} \phi_{n}\right\|^{2} \\
& +\left(1-\epsilon_{n} \bar{\gamma}\right)\left(1-\beta_{n}-\epsilon_{n} \bar{\gamma}\right)\left(1-\alpha_{n}\right) 2 \lambda_{n}\left\|y_{n}-W_{n} \phi_{n}\right\|\left\|B y_{n}-B p\right\| \\
& +\left(1-\epsilon_{n} \bar{\gamma}\right) \beta_{n}\left\|x_{n}-p\right\|^{2}+c_{n} \\
& =\left(1-\epsilon_{n} \bar{\gamma}\right)^{2}\left\|x_{n}-p\right\|^{2}-\left(1-\epsilon_{n} \bar{\gamma}\right)\left(1-\beta_{n}-\epsilon_{n} \bar{\gamma}\right)\left(1-\alpha_{n}\right)\left\|y_{n}-W_{n} \phi_{n}\right\|^{2} \\
& +\left(1-\epsilon_{n} \bar{\gamma}\right)\left(1-\beta_{n}-\epsilon_{n} \bar{\gamma}\right)\left(1-\alpha_{n}\right) 2 \lambda_{n}\left\|y_{n}-W_{n} \phi_{n}\right\|\left\|B y_{n}-B p\right\|+c_{n} \\
& \leq\left\|x_{n}-p\right\|^{2}-\left(1-\epsilon_{n} \bar{\gamma}\right)\left(1-\beta_{n}-\epsilon_{n} \bar{\gamma}\right)\left(1-\alpha_{n}\right)\left\|y_{n}-W_{n} \phi_{n}\right\|^{2} \\
& +\left(1-\epsilon_{n} \bar{\gamma}\right)\left(1-\beta_{n}-\epsilon_{n} \bar{\gamma}\right)\left(1-\alpha_{n}\right) 2 \lambda_{n}\left\|y_{n}-W_{n} \phi_{n}\right\|\left\|B y_{n}-B p\right\|+c_{n},
\end{aligned}
$$


which implies that

$$
\begin{aligned}
&\left(1-\epsilon_{n} \bar{\gamma}\right)\left(1-\beta_{n}-\epsilon_{n} \bar{\gamma}\right)\left(1-\alpha_{n}\right)\left\|y_{n}-W_{n} \phi_{n}\right\|^{2} \\
& \leq\left\|x_{n}-p\right\|^{2}-\left\|x_{n+1}-p\right\|^{2} \\
&+2\left(1-\epsilon_{n} \bar{\gamma}\right)\left(1-\beta_{n}-\epsilon_{n} \bar{\gamma}\right)\left(1-\alpha_{n}\right) \lambda_{n}\left\|y_{n}-W_{n} \phi_{n}\right\|\left\|B y_{n}-B p\right\|+c_{n} \\
& \leq\left\|x_{n}-x_{n+1}\right\|\left(\left\|x_{n}-p\right\|+\left\|x_{n+1}-p\right\|\right) \\
&+2\left(1-\epsilon_{n} \bar{\gamma}\right)\left(1-\beta_{n}-\epsilon_{n} \bar{\gamma}\right)\left(1-\alpha_{n}\right) \lambda_{n}\left\|y_{n}-W_{n} \phi_{n}\right\|\left\|B y_{n}-B p\right\|+c_{n}
\end{aligned}
$$

From (3.48) and (3.67), we obtain

$$
\lim _{n \rightarrow \infty}\left\|y_{n}-W_{n} \phi_{n}\right\|=0
$$

By using the same argument, we can prove that

$$
\lim _{n \rightarrow \infty}\left\|u_{n}-W_{n} \psi_{n}\right\|=0
$$

Note that

$$
\begin{aligned}
& k_{n}-W_{n} \phi_{n}=\alpha_{n}\left(x_{n}-W_{n} \phi_{n}\right), \\
& y_{n}-W_{n} \psi_{n}=\varphi_{n}\left(u_{n}-W_{n} \psi_{n}\right) .
\end{aligned}
$$

Since $\alpha_{n} \rightarrow 0$ and $\varphi_{n} \rightarrow 0$ as $n \rightarrow \infty$, respectively, we also have

$$
\lim _{n \rightarrow \infty}\left\|k_{n}-W_{n} \phi_{n}\right\|=\lim _{n \rightarrow \infty}\left\|y_{n}-W_{n} \psi_{n}\right\|=0
$$

On the other hand, we observe

$$
\left\|u_{n}-\theta_{n}\right\| \leq\left\|u_{n}-W_{n} \psi_{n}\right\|+\left\|W_{n} \psi_{n}-y_{n}\right\|+\left\|y_{n}-W_{n} \phi_{n}\right\|+\left\|W_{n} \phi_{n}-k_{n}\right\|+\left\|k_{n}-\theta_{n}\right\| .
$$

Applying (3.73), (3.83), (3.84), and (3.86), we have

$$
\lim _{n \rightarrow \infty}\left\|u_{n}-\theta_{n}\right\|=0
$$


On the other hand, we have

$$
\begin{aligned}
\left\|k_{n}-p\right\|^{2} & \leq \alpha_{n}\left\|x_{n}-p\right\|^{2}+\left(1-\alpha_{n}\right)\left\|W_{n} \phi_{n}-p\right\|^{2} \\
& \leq \alpha_{n}\left\|x_{n}-p\right\|^{2}+\left(1-\alpha_{n}\right)\left\|\phi_{n}-p\right\|^{2} \\
& \leq \alpha_{n}\left\|x_{n}-p\right\|^{2}+\left(1-\alpha_{n}\right)\left\|y_{n}-p\right\|^{2} \\
& \leq \alpha_{n}\left\|x_{n}-p\right\|^{2}+\left(1-\alpha_{n}\right)\left\{\varphi_{n}\left\|u_{n}-p\right\|^{2}+\left(1-\varphi_{n}\right)\left\|W_{n} \psi_{n}-p\right\|\right\} \\
& \leq \alpha_{n}\left\|x_{n}-p\right\|^{2}+\left(1-\alpha_{n}\right)\left\{\varphi_{n}\left\|u_{n}-p\right\|^{2}+\left(1-\varphi_{n}\right)\left\|\psi_{n}-p\right\|\right\} \\
& \leq \alpha_{n}\left\|x_{n}-p\right\|^{2}+\left(1-\alpha_{n}\right)\left\{\varphi_{n}\left\|u_{n}-p\right\|^{2}+\left(1-\varphi_{n}\right)\left\|u_{n}-p\right\|\right\} \\
& =\alpha_{n}\left\|x_{n}-p\right\|^{2}+\left(1-\alpha_{n}\right)\left\|u_{n}-p\right\|^{2} \\
& =\alpha_{n}\left\|x_{n}-p\right\|^{2}+\left(1-\alpha_{n}\right)\left\|T_{r_{n}}\left(I-r_{n} D\right) x_{n}-p\right\|^{2} \\
& \leq \alpha_{n}\left\|x_{n}-p\right\|^{2}+\left(1-\alpha_{n}\right)\left\|\left(I-r_{n} D\right) x_{n}-p\right\|^{2} \\
& \leq \alpha_{n}\left\|x_{n}-p\right\|^{2}+\left(1-\alpha_{n}\right)\left\{\left\|x_{n}-p\right\|^{2}-r_{n}\left(2 \eta-r_{n}\right)\left\|D x_{n}-D p\right\|^{2}\right\} \\
& =\left\|x_{n}-p\right\|^{2}-\left(1-\alpha_{n}\right) r_{n}\left(2 \eta-r_{n}\right)\left\|D x_{n}-D p\right\|^{2} .
\end{aligned}
$$

Substituting (3.89) into (3.64) and using conditions (C2) and (C7), we have

$$
\begin{aligned}
\left\|x_{n+1}-p\right\|^{2} \leq & \left(1-\epsilon_{n} \bar{\gamma}\right)\left(1-\beta_{n}-\epsilon_{n} \bar{\gamma}\right)\left\|k_{n}-p\right\|^{2}+\left(1-\epsilon_{n} \bar{\gamma}\right) \beta_{n}\left\|x_{n}-p\right\|^{2}+c_{n} \\
\leq & \left(1-\epsilon_{n} \bar{\gamma}\right)\left(1-\beta_{n}-\epsilon_{n} \bar{\gamma}\right)\left\{\left\|x_{n}-p\right\|^{2}-\left(1-\alpha_{n}\right) r_{n}\left(2 \eta-r_{n}\right)\left\|D x_{n}-D p\right\|^{2}\right\} \\
& +\left(1-\epsilon_{n} \bar{\gamma}\right) \beta_{n}\left\|x_{n}-p\right\|^{2}+c_{n} \\
= & \left(1-\epsilon_{n} \bar{\gamma}\right)\left(1-\beta_{n}-\epsilon_{n} \bar{\gamma}\right)\left(1-\alpha_{n}\right) r_{n}\left(2 \eta-r_{n}\right)\left\|D x_{n}-D p\right\|^{2} \\
& +\left(1-\epsilon_{n} \bar{\gamma}\right)^{2}\left\|x_{n}-p\right\|^{2}+c_{n} \\
\leq & \left\|x_{n}-p\right\|^{2}-\left(1-\alpha_{n}\right) r_{n}\left(2 \eta-r_{n}\right)\left\|D x_{n}-D p\right\| .
\end{aligned}
$$

This implies that

$$
\left(1-\alpha_{n}\right) r_{n}\left(2 \eta-r_{n}\right)\left\|D x_{n}-D p\right\| \leq\left\|x_{n}-p\right\|^{2}-\left\|x_{n+1}-p\right\|^{2}+c_{n}
$$

In view of the restrictions (C2) and (C7), we obtain that

$$
\lim _{n \rightarrow \infty}\left\|D x_{n}-D p\right\|=0
$$


Let $p \in \Theta:=\bigcap_{n=1}^{\infty} F\left(T_{n}\right) \cap \operatorname{EP}(F, D) \cap \operatorname{VI}(E, B)$. Since $u_{n}=T_{r_{n}}\left(x_{n}-r_{n} D x_{n}\right)$ and $T_{r_{n}}$ is firmly nonexpansive (Lemma 2.6), then we obtain

$$
\begin{aligned}
\left\|u_{n}-p\right\|^{2} & =\left\|T_{r_{n}}\left(x_{n}-r_{n} D x_{n}\right)-T_{r_{n}}\left(p-r_{n} D p\right)\right\|^{2} \\
& \leq\left\langle T_{r_{n}}\left(x_{n}-r_{n} D x_{n}\right)-T_{r_{n}}\left(p-r_{n} D p\right), u_{n}-p\right\rangle \\
& =\left\langle x_{n}-r_{n} D x_{n}-\left(p-r_{n} D p\right), u_{n}-p\right\rangle \\
& =\frac{1}{2}\left\{\left\|\left(x_{n}-r_{n} D x_{n}\right)-\left(p-r_{n} D p\right)\right\|^{2}+\left\|u_{n}-p\right\|^{2}\right. \\
& \left.\quad-\left\|\left(x_{n}-r_{n} D x_{n}\right)-\left(p-r_{n} D p\right)-\left(u_{n}-p\right)\right\|^{2}\right\} \\
& \leq \frac{1}{2}\left\{\left\|x_{n}-p\right\|^{2}+\left\|u_{n}-p\right\|^{2}-\left\|x_{n}-u_{n}-r_{n}\left(D x_{n}-D p\right)\right\|^{2}\right\} \\
& =\frac{1}{2}\left\{\left\|x_{n}-p\right\|^{2}+\left\|u_{n}-p\right\|^{2}-\left\|x_{n}-u_{n}\right\|^{2}+2 r_{n}\left\langle x_{n}-u_{n}, D x_{n}-D p\right\rangle\right. \\
& \left.\quad-r_{n}^{2}\left\|D x_{n}-D p\right\|^{2}\right\} .
\end{aligned}
$$

So, we obtain

$$
\left\|u_{n}-p\right\|^{2} \leq\left\|x_{n}-p\right\|^{2}-\left\|x_{n}-u_{n}\right\|^{2}+2 r_{n}\left\|x_{n}-u_{n}\right\|\left\|D x_{n}-D p\right\| .
$$

Therefore, we have

$$
\begin{aligned}
\left\|x_{n+1}-p\right\|^{2}= & \left(1-\epsilon_{n} \bar{\gamma}\right)\left(1-\beta_{n}-\epsilon_{n} \bar{\gamma}\right)\left\|W_{n} \theta_{n}-p\right\|^{2}+\left(1-\epsilon_{n} \bar{\gamma}\right) \beta_{n}\left\|x_{n}-p\right\|^{2}+c_{n} \\
\leq & \left(1-\epsilon_{n} \bar{\gamma}\right)\left(1-\beta_{n}-\epsilon_{n} \bar{\gamma}\right)\left\|\theta_{n}-p\right\|^{2}+\left(1-\epsilon_{n} \bar{\gamma}\right) \beta_{n}\left\|x_{n}-p\right\|^{2}+c_{n} \\
= & \left(1-\epsilon_{n} \bar{\gamma}\right)\left(1-\beta_{n}-\epsilon_{n} \bar{\gamma}\right)\left\|\left(\theta_{n}-u_{n}\right)+\left(u_{n}-p\right)\right\|^{2}+\left(1-\epsilon_{n} \bar{\gamma}\right) \beta_{n}\left\|x_{n}-p\right\|^{2}+c_{n} \\
\leq & \left(1-\epsilon_{n} \bar{\gamma}\right)\left(1-\beta_{n}-\epsilon_{n} \bar{\gamma}\right)\left\{\left\|\theta_{n}-u_{n}\right\|^{2}+\left\|u_{n}-p\right\|^{2}+2\left\langle\theta_{n}-u_{n}, u_{n}-p\right\rangle\right\} \\
& +\left(1-\epsilon_{n} \bar{\gamma}\right) \beta_{n}\left\|x_{n}-p\right\|^{2}+c_{n} \\
\leq & \left(1-\epsilon_{n} \bar{\gamma}\right)\left(1-\beta_{n}-\epsilon_{n} \bar{\gamma}\right)\left\|\theta_{n}-u_{n}\right\|^{2}+\left(1-\epsilon_{n} \bar{\gamma}\right)\left(1-\epsilon_{n} \bar{\gamma}-\beta_{n}\right)\left\|u_{n}-p\right\|^{2} \\
& +2\left(1-\epsilon_{n} \bar{\gamma}\right)\left(1-\beta_{n}-\epsilon_{n} \bar{\gamma}\right)\left\|\theta_{n}-u_{n}\right\|\left\|u_{n}-p\right\|+\left(1-\epsilon_{n} \bar{\gamma}\right) \beta_{n}\left\|x_{n}-p\right\|^{2}+c_{n} \\
\leq & \left(1-\epsilon_{n} \bar{\gamma}\right)\left(1-\beta_{n}-\epsilon_{n} \bar{\gamma}\right)\left\|\theta_{n}-u_{n}\right\|^{2} \\
& +\left(1-\epsilon_{n} \bar{\gamma}\right)\left(1-\beta_{n}-\epsilon_{n} \bar{\gamma}\right)\left\{\left\|x_{n}-p\right\|^{2}-\left\|x_{n}-u_{n}\right\|^{2}+2 r_{n}\left\|x_{n}-u_{n}\right\|\left\|D x_{n}-D p\right\|\right\} \\
& +2\left(1-\epsilon_{n} \bar{\gamma}\right)\left(1-\beta_{n}-\epsilon_{n} \bar{\gamma}\right)\left\|\theta_{n}-u_{n}\right\|\left\|u_{n}-p\right\|+\left(1-\epsilon_{n} \bar{\gamma}\right) \beta_{n}\left\|x_{n}-p\right\|^{2}+c_{n}
\end{aligned}
$$


Journal of Inequalities and Applications

$$
\begin{aligned}
= & \left(1-\epsilon_{n} \bar{\gamma}\right)\left(1-\beta_{n}-\epsilon_{n} \bar{\gamma}\right)\left\|\theta_{n}-u_{n}\right\|^{2}+\left(1-\epsilon_{n} \bar{\gamma}\right)\left(1-\beta_{n}-\epsilon_{n} \bar{\gamma}\right)\left\|x_{n}-p\right\|^{2} \\
& -\left(1-\epsilon_{n} \bar{\gamma}\right)\left(1-\beta_{n}-\epsilon_{n} \bar{\gamma}\right)\left\|x_{n}-u_{n}\right\|^{2} \\
& +\left(1-\epsilon_{n} \bar{\gamma}\right)\left(1-\beta_{n}-\epsilon_{n} \bar{\gamma}\right) 2 r_{n}\left\|x_{n}-u_{n}\right\|\left\|D x_{n}-D p\right\| \\
& +2\left(1-\epsilon_{n} \bar{\gamma}\right)\left(1-\beta_{n}-\epsilon_{n} \bar{\gamma}\right)\left\|\theta_{n}-u_{n}\right\|\left\|u_{n}-p\right\|+\left(1-\epsilon_{n} \bar{\gamma}\right) \beta_{n}\left\|x_{n}-p\right\|^{2}+c_{n} \\
= & \left(1-\epsilon_{n} \bar{\gamma}\right)^{2}\left\|x_{n}-p\right\|^{2}-\left(1-\epsilon_{n} \bar{\gamma}\right)\left(1-\beta_{n}-\epsilon_{n} \bar{\gamma}\right)\left\|x_{n}-u_{n}\right\|^{2} \\
& +\left(1-\epsilon_{n} \bar{\gamma}\right)\left(1-\beta_{n}-\epsilon_{n} \bar{\gamma}\right)\left\|\theta_{n}-u_{n}\right\|^{2} \\
& +\left(1-\epsilon_{n} \bar{\gamma}\right)\left(1-\beta_{n}-\epsilon_{n} \bar{\gamma}\right) 2 r_{n}\left\|x_{n}-u_{n}\right\|\left\|D x_{n}-D p\right\| \\
& +2\left(1-\epsilon_{n} \bar{\gamma}\right)\left(1-\beta_{n}-\epsilon_{n} \bar{\gamma}\right)\left\|\theta_{n}-u_{n}\right\|\left\|u_{n}-p\right\|+c_{n} \\
= & \left(1-2 \epsilon_{n} \bar{\gamma}+\left(\epsilon_{n} \bar{\gamma}\right)^{2}\right)\left\|x_{n}-p\right\|^{2}-\left(1-\epsilon_{n} \bar{\gamma}\right)\left(1-\beta_{n}-\epsilon_{n} \bar{\gamma}\right)\left\|x_{n}-u_{n}\right\|^{2} \\
& +\left(1-\epsilon_{n} \bar{\gamma}\right)\left(1-\beta_{n}-\epsilon_{n} \bar{\gamma}\right)\left\|\theta_{n}-u_{n}\right\|^{2} \\
& +\left(1-\epsilon_{n} \bar{\gamma}\right)\left(1-\beta_{n}-\epsilon_{n} \bar{\gamma}\right) 2 r_{n}\left\|x_{n}-u_{n}\right\|\left\|D x_{n}-D p\right\| \\
& +2\left(1-\epsilon_{n} \bar{\gamma}\right)\left(1-\beta_{n}-\epsilon_{n} \bar{\gamma}\right)\left\|\theta_{n}-u_{n}\right\|\left\|u_{n}-p\right\|+c_{n} \\
\leq & \left\|x_{n}-p\right\|^{2}+\left(\epsilon_{n} \bar{\gamma}\right)^{2}\left\|x_{n}-p\right\|^{2}+\left(1-\epsilon_{n} \bar{\gamma}\right)\left(1-\beta_{n}-\epsilon_{n} \bar{\gamma}\right)\left\|\theta_{n}-u_{n}\right\|^{2} \\
& +\left(1-\epsilon_{n} \bar{\gamma}\right)\left(1-\beta_{n}-\epsilon_{n} \bar{\gamma}\right)\left\|x_{n}-u_{n}\right\|^{2} \\
& +\left(1-\epsilon_{n} \bar{\gamma}\right)\left(1-\beta_{n}-\epsilon_{n} \bar{\gamma}\right) 2 r_{n}\left\|x_{n}-u_{n}\right\|\left\|D x_{n}-D p\right\| \\
& +2\left(1-\epsilon_{n} \bar{\gamma}\right)\left(1-\beta_{n}-\epsilon_{n} \bar{\gamma}\right)\left\|\theta_{n}-u_{n}\right\|\left\|u_{n}-p\right\|+c_{n} . \\
& +(3 .)
\end{aligned}
$$

It follows that

$$
\begin{aligned}
&\left(1-\epsilon_{n} \bar{\gamma}\right)\left(1-\beta_{n}-\epsilon_{n} \bar{\gamma}\right)\left\|x_{n}-u_{n}\right\|^{2} \\
& \leq\left\|x_{n}-p\right\|^{2}-\left\|x_{n+1}-p\right\|^{2}+\left(\epsilon_{n} \bar{\gamma}\right)^{2}\left\|x_{n}-p\right\|^{2} \\
&+\left(1-\epsilon_{n} \bar{\gamma}\right)\left(1-\beta_{n}-\epsilon_{n} \bar{\gamma}\right)\left\|\theta_{n}-u_{n}\right\|^{2}+\left(1-\epsilon_{n} \bar{\gamma}\right)\left(1-\beta_{n}-\epsilon_{n} \bar{\gamma}\right) 2 r_{n}\left\|x_{n}-u_{n}\right\|\left\|D x_{n}-D p\right\| \\
&+2\left(1-\epsilon_{n} \bar{\gamma}\right)\left(1-\beta_{n}-\epsilon_{n} \bar{\gamma}\right)\left\|\theta_{n}-u_{n}\right\|\left\|u_{n}-p\right\|+c_{n} \\
& \leq\left\|x_{n}-x_{n+1}\right\|\left(\left\|x_{n}-p\right\|+\left\|x_{n+1}-p\right\|\right)+\left(\epsilon_{n} \bar{\gamma}\right)^{2}\left\|x_{n}-p\right\|^{2} \\
&+\left(1-\epsilon_{n} \bar{\gamma}\right)\left(1-\beta_{n}-\epsilon_{n} \bar{\gamma}\right)\left\|\theta_{n}-u_{n}\right\|^{2}+\left(1-\epsilon_{n} \bar{\gamma}\right)\left(1-\beta_{n}-\epsilon_{n} \bar{\gamma}\right) 2 r_{n}\left\|x_{n}-u_{n}\right\|\left\|D x_{n}-D p\right\| \\
&+2\left(1-\epsilon_{n} \bar{\gamma}\right)\left(1-\beta_{n}-\epsilon_{n} \bar{\gamma}\right)\left\|\theta_{n}-u_{n}\right\|\left\|u_{n}-p\right\|+c_{n} .
\end{aligned}
$$

Using $\epsilon_{n} \rightarrow 0, c_{n} \rightarrow 0$ as $n \rightarrow \infty$, (3.48), (3.88), and (3.92), we obtain

$$
\lim _{n \rightarrow \infty}\left\|x_{n}-u_{n}\right\|=0
$$


Since $\liminf \operatorname{in}_{n \rightarrow \infty} r_{n}>0$, we obtain

$$
\lim _{n \rightarrow \infty}\left\|\frac{x_{n}-u_{n}}{r_{n}}\right\|=\lim _{n \rightarrow \infty} \frac{1}{r_{n}}\left\|x_{n}-u_{n}\right\|=0 .
$$

Note that

$$
\left\|x_{n}-\theta_{n}\right\| \leq\left\|x_{n}-u_{n}\right\|+\left\|u_{n}-\theta_{n}\right\|,
$$

and thus from (3.88) and (3.97), we have

$$
\lim _{n \rightarrow \infty}\left\|x_{n}-\theta_{n}\right\|=0
$$

Observe that

$$
\left\|W_{n} \theta_{n}-\theta_{n}\right\| \leq\left\|W_{n} \theta_{n}-x_{n}\right\|+\left\|x_{n}-\theta_{n}\right\| .
$$

Applying (3.53) and (3.100), we obtain

$$
\lim _{n \rightarrow \infty}\left\|W_{n} \theta_{n}-\theta_{n}\right\|=0 .
$$

Let $W$ be the mapping defined by (2.11). Since $\left\{\theta_{n}\right\}$ is bounded, applying Lemma 2.10 and (3.102), we have

$$
\left\|W \theta_{n}-\theta_{n}\right\| \leq\left\|W \theta_{n}-W_{n} \theta_{n}\right\|+\left\|W_{n} \theta_{n}-\theta_{n}\right\| \longrightarrow 0 \text { as } n \longrightarrow \infty
$$

Step 5. We claim that $\lim \sup _{n \rightarrow \infty}\left\langle(A-\gamma f) z, z-x_{n}\right\rangle \leq 0$, where $z$ is the unique solution of the variational inequality $\langle(A-\gamma f) z, x-z\rangle \geq 0$, for all $x \in \Theta$.

Since $z=P_{\Theta}(I-A+\gamma f)(z)$ is a unique solution of the variational inequality (3.5), to show this inequality, we choose a subsequence $\left\{\theta_{n_{i}}\right\}$ of $\left\{\theta_{n}\right\}$ such that

$$
\lim _{i \rightarrow \infty}\left\langle(A-\gamma f) z, z-\theta_{n_{i}}\right\rangle=\limsup _{n \rightarrow \infty}\left\langle(A-\gamma f) z, z-\theta_{n}\right\rangle
$$

Since $\left\{\theta_{n_{i}}\right\}$ is bounded, there exists a subsequence $\left\{\theta_{n_{i_{j}}}\right\}$ of $\left\{\theta_{n_{i}}\right\}$ which converges weakly to $w \in E$. Without loss of generality, we can assume that $\theta_{n_{i}} \rightarrow w$. From $\left\|W \theta_{n}-\theta_{n}\right\| \rightarrow 0$, we obtain $W \theta_{n_{i}} \rightarrow w$. Next, We show that $w \in \Theta$, where $\Theta:=\bigcap_{n=1}^{\infty} F\left(T_{n}\right) \cap \operatorname{EP}(F, D) \cap \operatorname{VI}(E, B)$.

(a) First, we prove $w \in \operatorname{EP}(F, D)$.

Since $u_{n}=T_{r_{n}}\left(x_{n}-r_{n} D x_{n}\right)$, we know that

$$
F\left(u_{n}, y\right)+\left\langle D x_{n}, y-u_{n}\right\rangle+\frac{1}{r_{n}}\left\langle y-u_{n}, u_{n}-x_{n}\right\rangle \geq 0, \quad \forall y \in E
$$


From (A2), we also have

$$
\left\langle D x_{n}, y-u_{n}\right\rangle+\frac{1}{r_{n}}\left\langle y-u_{n}, u_{n}-x_{n}\right\rangle \geq-F\left(u_{n}, y\right) \geq F\left(y, u_{n}\right)
$$

Replacing $n$ by $n_{i}$, we have

$$
\left\langle D x_{n_{i}}, y-u_{n_{i}}\right\rangle+\left\langle y-u_{n_{i}}, \frac{u_{n_{i}}-x_{n_{i}}}{r_{n_{i}}}\right\rangle \geq F\left(y, u_{n_{i}}\right)
$$

For any $t$ with $0<t \leq 1$ and $y \in E$, let $\varphi_{t}=t y+(1-t) z$. Since $y \in E$ and $z \in E$, we have $\varphi_{t} \in E$. So, from (3.107) we have

$$
\begin{aligned}
\left\langle\varphi_{t}-u_{n_{i}}, D \varphi_{t}\right\rangle \geq & \left\langle\varphi_{t}-u_{n_{i}}, D \varphi_{t}\right\rangle-\left\langle D x_{n_{i}}, \varphi_{t}-u_{n_{i}}\right\rangle-\left\langle\varphi_{t}-u_{n_{i}}, \frac{u_{n_{i}}-x_{n_{i}}}{r_{n_{i}}}\right\rangle+F\left(\varphi_{t}, u_{n_{i}}\right) \\
\geq & \left\langle\varphi_{t}-u_{n_{i}}, D \varphi_{t}-D u_{n_{i}}\right\rangle+\left\langle\varphi_{t}-u_{n_{i}}, D u_{n_{i}}-D x_{n_{i}}\right\rangle \\
& -\left\langle\varphi_{t}-u_{n_{i}}, \frac{u_{n_{i}}-x_{n_{i}}}{r_{n_{i}}}\right\rangle+F\left(\varphi_{t}, u_{n_{i}}\right) .
\end{aligned}
$$

Since $D$ is Lipschitz continuous, from (3.97), we have $\left\|D u_{n_{i}}-D x_{n_{i}}\right\| \rightarrow 0$ as $i \rightarrow \infty$.

Further, from the monotonicity of $D$, we get that

$$
\left\langle\varphi_{t}-u_{n_{i}}, D \varphi_{t}-D u_{n_{i}}\right\rangle \geq 0 .
$$

It follows from (A4) and (3.108) that

$$
\left\langle\varphi_{t}-z, D \varphi_{t}\right\rangle \geq F\left(\varphi_{t}, z\right) .
$$

From (A1), (A4), and (3.110), we also have

$$
\begin{aligned}
0=F\left(\varphi_{t}, \varphi_{t}\right) & \leq t F\left(\varphi_{t}, y\right)+(1-t) F\left(\varphi_{t}, z\right) \\
& \leq t F\left(\varphi_{t}, y\right)+(1-t)\left\langle\varphi_{t}-z, D \varphi_{t}\right) \\
& =t F\left(\varphi_{t}, y\right)+(1-t) t\left\langle y-z, D \varphi_{t}\right),
\end{aligned}
$$

and hence

$$
F\left(\varphi_{t}, y\right)+(1-t)\left\langle y-z, D \varphi_{t}\right) \geq 0 .
$$


Letting $t \rightarrow \infty$ in the above inequality, we have, for each $y \in E$,

$$
F(z, y)+\langle y-z, D z) \geq 0
$$

Thus $z \in \operatorname{EP}(F, D)$.

(b) Next, we show that $w \in \bigcap_{n=1}^{\infty} F\left(T_{n}\right)$.

By Lemma 2.9, we have $F(W)=\bigcap_{n=1}^{\infty} F\left(T_{n}\right)$. Assume $w \notin F(W)$. Since $\left\|x_{n}-\theta_{n}\right\| \rightarrow$ 0 , we know that $\theta_{n_{i}} \rightarrow w(i \rightarrow \infty)$ and $w \neq W w$, and it follows by the Opial's condition (Lemma 2.3) that

$$
\begin{aligned}
\liminf _{i \rightarrow \infty}\left\|\theta_{n_{i}}-w\right\| & <\liminf _{i \rightarrow \infty}\left\|\theta_{n_{i}}-W w\right\| \\
& \leq \liminf _{i \rightarrow \infty}\left(\left\|\theta_{n_{i}}-W \theta_{n_{i}}\right\|+\left\|W \theta_{n_{i}}-W w\right\|\right) \\
& <\liminf _{i \rightarrow \infty}\left\|\theta_{n_{i}}-w\right\|,
\end{aligned}
$$

that is a contradiction. Thus, we have $w \in F(W)=\bigcap_{n=1}^{\infty} F\left(T_{n}\right)$.

(c) Finally, Now we prove that $w \in \operatorname{VI}(E, B)$. Define,

$$
T w_{1}= \begin{cases}B w_{1}+N_{E} w_{1}, & \text { if } w_{1} \in E \\ \emptyset, & \text { if } w_{1} \notin E .\end{cases}
$$

Since $B$ is relaxed $(u, v)$-cocoercive and condition (C6), we have

$$
\langle B x-B y, x-y\rangle \geq(-u)\|B x-B y\|^{2}+v\|x-y\|^{2} \geq\left(v-u \xi^{2}\right)\|x-y\|^{2} \geq 0,
$$

which yields that $B$ is monotone. Then, $T$ is maximal monotone. Let $\left(w_{1}, w_{2}\right) \in G(T)$. Since $w_{2}-B w_{1} \in N_{E} w_{1}$ and $\theta_{n} \in E$, we have $\left\langle w_{1}-\theta_{n}, w_{2}-B w_{1}\right\rangle \geq 0$. On the other hand, from $\theta_{n}=P_{E}\left(k_{n}-\tau_{n} B k_{n}\right)$, we have

$$
\left\langle w_{1}-\theta_{n}, \theta_{n}-\left(k_{n}-\tau_{n} B k_{n}\right)\right\rangle \geq 0,
$$

and hence

$$
\left\langle w_{1}-\theta_{n}, \frac{\left(\theta_{n}-k_{n}\right)}{\tau_{n}}+B k_{n}\right\rangle \geq 0 .
$$


Therefore, we have

$$
\begin{aligned}
\left\langle w_{1}-\theta_{n_{i}}, w\right\rangle & \geq\left\langle w_{1}-\theta_{n_{i}}, B w_{1}\right\rangle \\
& \geq\left\langle w_{1}-\theta_{n_{i}}, B w_{1}\right\rangle-\left\langle w_{1}-\theta_{n_{i}}, \frac{\left(\theta_{n_{i}}-k_{n_{i}}\right)}{\tau_{n_{i}}}+B k_{n_{i}}\right\rangle \\
& =\left\langle w_{1}-\theta_{n_{i}}, B w_{1}-B k_{n_{i}}-\frac{\left(\theta_{n_{i}}-k_{n_{i}}\right)}{\tau_{n_{i}}}\right\rangle \\
& =\left\langle w_{1}-\theta_{n_{i}}, B v-B \theta_{n_{i}}\right\rangle+\left\langle w_{1}-\theta_{n_{i}}, B \theta_{n_{i}}-B k_{n_{i}}\right\rangle-\left\langle w_{1}-\theta_{n_{i}}, \frac{\left(\theta_{n_{\mathrm{i}}}-k_{n_{i}}\right)}{\tau_{n_{i}}}\right\rangle \\
& \geq\left\langle w_{1}-\theta_{n_{i}}, B \theta_{n_{i}}-B k_{n_{i}}\right\rangle-\left\langle w_{1}-\theta_{n_{i}}, \frac{\left(\theta_{n_{i}}-k_{n_{i}}\right)}{\tau_{n_{i}}}\right\rangle,
\end{aligned}
$$

which implies that

$$
\left\langle w_{1}-w, w_{2}\right\rangle \geq 0
$$

Since $T$ is maximal monotone, we have $w \in T^{-1} 0$ and hence $w \in \operatorname{VI}(E, B)$. That is, $w \in \Theta$, where $\Theta:=\bigcap_{n=1}^{\infty} F\left(T_{n}\right) \cap \operatorname{EP}(F, D) \cap \operatorname{VI}(E, B)$. Since $z=P_{\Theta}(I-A+\gamma f)(z)$, it follows that

$$
\begin{aligned}
\limsup _{n \rightarrow \infty}\left\langle(A-\gamma f) z, z-x_{n}\right\rangle & =\limsup _{n \rightarrow \infty}\left\langle(A-\gamma f) z, z-\theta_{n}\right\rangle \\
& =\lim _{i \rightarrow \infty}\left\langle(A-\gamma f) z, z-\theta_{n_{i}}\right\rangle \\
& =\langle(A-\gamma f) z, z-w\rangle \leq 0 .
\end{aligned}
$$

On the other hand, we have

$$
\begin{aligned}
\left\langle(A-\gamma f) z, z-W_{n} \theta_{n}\right\rangle & =\left\langle(A-\gamma f) z, x_{n}-W_{n} \theta_{n}\right\rangle+\left\langle(A-\gamma f) z, z-x_{n}\right\rangle \\
& \leq\|(A-\gamma f) z\|\left\|x_{n}-W_{n} \theta_{n}\right\|+\left\langle(A-\gamma f) z, z-x_{n}\right\rangle .
\end{aligned}
$$

From (3.53) and (3.121), we obtain that

$$
\limsup _{n \rightarrow \infty}\left\langle\gamma f(z)-A z, W_{n} \theta_{n}-z\right\rangle \leq 0 .
$$

Step 6. Finally, we show that $\left\{x_{n}\right\}$ and $\left\{u_{n}\right\}$ converge strongly to $z=P_{\Theta}(I-A+\gamma f)(z)$. 
Indeed, from (3.4) and Lemma 2.4, we obtain

$$
\begin{aligned}
& \left\|x_{n+1}-z\right\|^{2}=\left\|\epsilon_{n} \gamma f\left(W_{n} x_{n}\right)+\beta_{n} x_{n}+\left(\left(1-\beta_{n}\right) I-\epsilon_{n} A\right) W_{n} \theta_{n}-z\right\|^{2} \\
& =\left\|\left(\left(1-\beta_{n}\right) I-\epsilon_{n} A\right)\left(W_{n} \theta_{n}-z\right)+\beta_{n}\left(x_{n}-z\right)+\epsilon_{n}\left(\gamma f\left(W_{n} x_{n}\right)-A z\right)\right\|^{2} \\
& =\left\|\left(\left(1-\beta_{n}\right) I-\epsilon_{n} A\right)\left(W_{n} \theta_{n}-z\right)+\beta_{n}\left(x_{n}-z\right)\right\|^{2}+\epsilon_{n}^{2}\left\|\gamma f\left(W_{n} x_{n}\right)-A z\right\|^{2} \\
& +2 \beta_{n} \epsilon_{n}\left\langle x_{n}-z, \gamma f\left(W_{n} x_{n}\right)-A z\right\rangle \\
& +2 \epsilon_{n}\left\langle\left(\left(1-\beta_{n}\right) I-\epsilon_{n} A\right)\left(W_{n} \theta_{n}-z\right), \gamma f\left(W_{n} x_{n}\right)-A z\right\rangle \\
& \leq\left(\left(1-\beta_{n}-\epsilon_{n} \bar{\gamma}\right)\left\|W_{n} \theta_{n}-z\right\|+\beta_{n}\left\|x_{n}-z\right\|\right)^{2}+\epsilon_{n}^{2}\left\|\gamma f\left(W_{n} x_{n}\right)-A z\right\|^{2} \\
& +2 \beta_{n} \epsilon_{n} \gamma\left\langle x_{n}-z, f\left(W_{n} x_{n}\right)-f(z)\right\rangle+2 \beta_{n} \epsilon_{n}\left\langle x_{n}-z, \gamma f(z)-A z\right\rangle \\
& +2\left(1-\beta_{n}\right) \gamma \epsilon_{n}\left\langle W_{n} \theta_{n}-z, f\left(W_{n} x_{n}\right)-f(z)\right\rangle \\
& +2\left(1-\beta_{n}\right) \epsilon_{n}\left\langle W_{n} \theta_{n}-z, \gamma f(z)-A z\right\rangle-2 \epsilon_{n}^{2}\left\langle A\left(W_{n} \theta_{n}-z\right), \gamma f(z)-A z\right\rangle, \\
& \leq\left(\left(1-\beta_{n}-\epsilon_{n} \bar{\gamma}\right)\left\|W_{n} \theta_{n}-z\right\|+\beta_{n}\left\|x_{n}-z\right\|\right)^{2}+\epsilon_{n}^{2}\left\|\gamma f\left(W_{n} x_{n}\right)-A z\right\|^{2} \\
& +2 \beta_{n} \epsilon_{n} \gamma\left\|x_{n}-z\right\|\left\|f\left(W_{n} x_{n}\right)-f(z)\right\|+2 \beta_{n} \epsilon_{n}\left\langle x_{n}-z, \gamma f(z)-A z\right\rangle \\
& +2\left(1-\beta_{n}\right) \gamma \epsilon_{n}\left\|W_{n} \theta_{n}-z\right\|\left\|f\left(W_{n} x_{n}\right)-f(z)\right\| \\
& +2\left(1-\beta_{n}\right) \epsilon_{n}\left\langle W_{n} \theta_{n}-z, \gamma f(z)-A z\right\rangle-2 \epsilon_{n}^{2}\left\langle A\left(W_{n} \theta_{n}-z\right), \gamma f(z)-A z\right\rangle, \\
& \leq\left(\left(1-\beta_{n}-\epsilon_{n} \bar{\gamma}\right)\left\|\theta_{n}-z\right\|+\beta_{n}\left\|x_{n}-z\right\|\right)^{2}+\epsilon_{n}^{2}\left\|\gamma f\left(W_{n} x_{n}\right)-A z\right\|^{2} \\
& +2 \beta_{n} \epsilon_{n} \gamma\left\|x_{n}-z\right\|\left\|f\left(W_{n} x_{n}\right)-f(z)\right\|+2 \beta_{n} \epsilon_{n}\left\langle x_{n}-z, \gamma f(z)-A z\right\rangle \\
& +2\left(1-\beta_{n}\right) \gamma \epsilon_{n}\left\|\theta_{n}-z\right\|\left\|f\left(W_{n} x_{n}\right)-f(z)\right\|+2\left(1-\beta_{n}\right) \epsilon_{n}\left\langle W_{n} \theta_{n}-z, \gamma f(z)-A z\right\rangle \\
& -2 \epsilon_{n}^{2}\left\langle A\left(W_{n} \theta_{n}-z\right), \gamma f(z)-A z\right\rangle \\
& \leq\left(\left(1-\beta_{n}-\epsilon_{n} \bar{\gamma}\right)\left\|x_{n}-z\right\|+\beta_{n}\left\|x_{n}-z\right\|\right)^{2}+\epsilon_{n}^{2}\left\|\gamma f\left(W_{n} x_{n}\right)-A z\right\|^{2} \\
& +2 \beta_{n} \epsilon_{n} \gamma \alpha\left\|x_{n}-z\right\|^{2}+2 \beta_{n} \epsilon_{n}\left\langle x_{n}-z, \gamma f(z)-A z\right\rangle \\
& +2\left(1-\beta_{n}\right) \gamma \epsilon_{n} \alpha\left\|x_{n}-z\right\|^{2} \\
& +2\left(1-\beta_{n}\right) \epsilon_{n}\left\langle W_{n} \theta_{n}-z, \gamma f(z)-A z\right\rangle-2 \epsilon_{n}^{2}\left\langle A\left(W_{n} \theta_{n}-z\right), \gamma f(z)-A z\right\rangle \\
& =\left[\left(1-\epsilon_{n} \bar{\gamma}\right)^{2}+2 \beta_{n} \epsilon_{n} \gamma \alpha+2\left(1-\beta_{n}\right) \gamma \epsilon_{n} \alpha\right]\left\|x_{n}-z\right\|^{2}+\epsilon_{n}^{2}\left\|\gamma f\left(W_{n} x_{n}\right)-A z\right\|^{2} \\
& +2 \beta_{n} \epsilon_{n}\left\langle x_{n}-z, \gamma f(z)-A z\right\rangle+2\left(1-\beta_{n}\right) \epsilon_{n}\left\langle W_{n} \theta_{n}-z, \gamma f(z)-A z\right\rangle \\
& -2 \epsilon_{n}^{2}\left\langle A\left(W_{n} \theta_{n}-z\right), \gamma f(z)-A z\right\rangle \\
& \leq\left[1-2(\bar{\gamma}-\alpha \gamma) \epsilon_{n}\right]\left\|x_{n}-z\right\|^{2}+\bar{\gamma}^{2} \epsilon_{n}^{2}\left\|x_{n}-z\right\|^{2}+\epsilon_{n}^{2}\left\|\gamma f\left(W_{n} x_{n}\right)-A z\right\|^{2} \\
& +2 \beta_{n} \epsilon_{n}\left\langle x_{n}-z, \gamma f(z)-A z\right\rangle+2\left(1-\beta_{n}\right) \epsilon_{n}\left\langle W_{n} \theta_{n}-z, \gamma f(z)-A z\right\rangle \\
& +2 \epsilon_{n}^{2}\left\|A\left(W_{n} \theta_{n}-z\right)\right\|\|\gamma f(z)-A z\|
\end{aligned}
$$


Journal of Inequalities and Applications

$$
\begin{aligned}
=\left[1-2(\bar{\gamma}-\alpha \gamma) \epsilon_{n}\right]\left\|x_{n}-z\right\|^{2} \\
+\epsilon_{n}\left\{\epsilon_{n}\left(\bar{\gamma}^{2}\left\|x_{n}-z\right\|^{2}+\left\|\gamma f\left(\mathrm{~W}_{n} x_{n}\right)-A z\right\|^{2}+2\left\|A\left(W_{n} \theta_{n}-z\right)\right\|\|\gamma f(z)-A z\|\right)\right. \\
\left.\quad+2 \beta_{n}\left\langle x_{n}-z, \gamma f(z)-A z\right\rangle+2\left(1-\beta_{n}\right)\left\langle W_{n} \theta_{n}-z, \gamma f(z)-A z\right\rangle\right\} .
\end{aligned}
$$

Since $\left\{x_{n}\right\},\left\{f\left(W_{n} x_{n}\right)\right\}$, and $\left\{W_{n} \theta_{n}\right\}$ are bounded, we can take a constant $M>0$ such that

$$
\bar{\gamma}^{2}\left\|x_{n}-z\right\|^{2}+\left\|\gamma f\left(W_{n} x_{n}\right)-A z\right\|^{2}+2\left\|A\left(W_{n} \theta_{n}-z\right)\right\|\|\gamma f(z)-A z\| \leq M
$$

for all $n \geq 0$. It then follows that

$$
\left\|x_{n+1}-z\right\|^{2} \leq\left(1-l_{n}\right)\left\|x_{n}-z\right\|^{2}+\epsilon_{n} \sigma_{n}
$$

where

$$
\begin{gathered}
l_{n}=2(\bar{\gamma}-\alpha \gamma) \epsilon_{n} \\
\sigma_{n}=\epsilon_{n} M+2 \beta_{n}\left\langle x_{n}-z, \gamma f(z)-A z\right\rangle+2\left(1-\beta_{n}\right)\left\langle W_{n} \theta_{n}-z, \gamma f(z)-A z\right\rangle .
\end{gathered}
$$

Using (C1), (3.121), and (3.123), we get $l_{n} \rightarrow 0, \sum_{n=1}^{\infty} l_{n}=\infty$ and $\lim \sup _{n \rightarrow \infty}\left(\sigma_{n} / l_{n}\right) \leq 0$. Applying Lemma 2.13 to (3.126), we conclude that $x_{n} \rightarrow z$ in norm. Finally, noticing $\| u_{n}-$ $z\|=\| T_{r_{n}}\left(x_{n}-r_{n} D x_{n}\right)-T_{r_{n}}\left(z-r_{n} D z\right)\|\leq\| x_{n}-z \|$, we also conclude that $u_{n} \rightarrow z$ in norm. This completes the proof.

Corollary 3.4. Let $E$ be a nonempty closed convex subset of a real Hilbert space $H$. Let $F: E \times E \rightarrow \mathbb{R}$ be a bifunction satisfying $(A 1)-(A 4)$, let $B: E \rightarrow H$ be relaxed $(u, v)$-cocoercive and $\xi$-Lipschitz continuous mappings, and let $\left\{T_{n}\right\}$ be an infinite family of nonexpansive mappings of $E$ into itself such that $\Theta:=\bigcap_{n=1}^{\infty} F\left(T_{n}\right) \cap \mathrm{EP}(F) \cap \operatorname{VI}(E, B) \neq \emptyset$. Let $f$ be a contraction mapping of $E$ into itself with $\alpha \in(0,1)$. Let $\left\{x_{n}\right\},\left\{y_{n}\right\},\left\{k_{n}\right\}$, and $\left\{u_{n}\right\}$ be sequences generated by

$$
\begin{gathered}
x_{1} \in E \text { chosen arbitrary, } \\
F\left(u_{n}, y\right)+\frac{1}{r_{n}}\left\langle y-u_{n}, u_{n}-x_{n}\right\rangle \geq 0, \quad \forall y \in E, \\
y_{n}=\varphi_{n} u_{n}+\left(1-\varphi_{n}\right) W_{n} P_{E}\left(u_{n}-\delta_{n} B u_{n}\right), \\
k_{n}=\alpha_{n} x_{n}+\left(1-\alpha_{n}\right) W_{n} P_{E}\left(y_{n}-\lambda_{n} B y_{n}\right), \\
x_{n+1}=\epsilon_{n} f\left(W_{n} x_{n}\right)+\beta_{n} x_{n}+\gamma_{n} W_{n} P_{E}\left(k_{n}-\tau_{n} B k_{n}\right), \quad \forall n \geq 1,
\end{gathered}
$$

where $\left\{W_{n}\right\}$ is the sequence generated by (1.24) and $\left\{\epsilon_{n}\right\},\left\{\alpha_{n}\right\},\left\{\varphi_{n}\right\}$, and $\left\{\beta_{n}\right\}$ are sequences in $(0,1)$ and $\left\{r_{n}\right\}$ is a real sequence in $(0, \infty)$ satisfying the following conditions:

(C1) $\epsilon_{n}+\beta_{n}+\gamma_{n}=1$,

(C2) $\lim _{n \rightarrow \infty} \epsilon_{n}=0, \sum_{n=1}^{\infty} \epsilon_{n}=\infty$, 
(C4) $\lim _{n \rightarrow \infty} \alpha_{n}=\lim _{n \rightarrow \infty} \varphi_{n}=0$,

(C5) $0<\liminf _{n \rightarrow \infty} \beta_{n} \leq \lim \sup _{n \rightarrow \infty} \beta_{n}<1$,

(C6) $\lim _{n \rightarrow \infty}\left|\lambda_{n+1}-\lambda_{n}\right|=\lim _{n \rightarrow \infty}\left|\delta_{n+1}-\delta_{n}\right|=\lim _{n \rightarrow \infty}\left|\tau_{n+1}-\tau_{n}\right|=0$,

(C7) $\left\{\tau_{n}\right\},\left\{\lambda_{n}\right\},\left\{\delta_{n}\right\} \subset[a, b]$ for some $a, b$ with $0 \leq a \leq b \leq 2\left(v-u \xi^{2}\right) / \xi^{2}, v>u \xi^{2}$.

Then, $\left\{x_{n}\right\}$ and $\left\{u_{n}\right\}$ converge strongly to a point $z \in \Theta$, where $z=P_{\Theta} f(z)$.

Proof. Put $A=I, \gamma \equiv 1, \gamma_{n}=1-\epsilon_{n}-\beta_{n}, D=0$ (:the zero mapping) and $\left\{\epsilon_{n}\right\}=0$ in Theorem 3.3. Then $y_{n}=v_{n}=u_{n}$, and for any $\eta>0$, we see that

$$
\langle D x-D y, x-y\rangle \geq \eta\|D x-D y\|^{2}, \quad \forall x, y \in E .
$$

Let $\left\{r_{n}\right\}$ be a sequence satisfying the restriction: $c \leq r_{n} \leq d$, where $c, d \in(0, \infty)$. Then we can obtain the desired conclusion easily from Theorem 3.3.

Corollary 3.5. Let $E$ be a nonempty closed convex subset of a real Hilbert space $H$. Let $\left\{T_{n}\right\}$ be an infinite family of nonexpansive mappings of $E$ into itself and let $B: E \rightarrow H$ be relaxed $(u, v)$ cocoercive and $\xi$-Lipschitz continuous mappings such that $\Theta:=\bigcap_{n=1}^{\infty} F\left(T_{n}\right) \cap \operatorname{VI}(E, B) \neq \emptyset$. Let $f$ : $E \rightarrow E$ be a contraction mapping with $0<\alpha<1$ and let $A$ be a strongly positive linear bounded operator on $H$ with coefficient $\bar{\gamma}>0$ and $0<\gamma<\bar{\gamma} / \alpha$. Let $\left\{x_{n}\right\},\left\{y_{n}\right\}$, and $\left\{k_{n}\right\}$ be sequences generated by

$$
\begin{gathered}
x_{1} \in E \text { chosen arbitrary, } \\
y_{n}=\varphi_{n} x_{n}+\left(1-\varphi_{n}\right) W_{n} P_{E}\left(x_{n}-\delta_{n} B x_{n}\right), \\
k_{n}=\alpha_{n} x_{n}+\left(1-\alpha_{n}\right) W_{n} P_{E}\left(y_{n}-\lambda_{n} B y_{n}\right), \\
x_{n+1}=\epsilon_{n} \gamma f\left(W_{n} x_{n}\right)+\beta_{n} x_{n}+\left(\left(1-\beta_{n}\right) I-\epsilon_{n} A\right) W_{n} P_{E}\left(k_{n}-\tau_{n} B k_{n}\right), \quad \forall n \geq 1,
\end{gathered}
$$

where $\left\{W_{n}\right\}$ is the sequence generated by (1.24) and $\left\{\epsilon_{n}\right\},\left\{\alpha_{n}\right\},\left\{\varphi_{n}\right\}$, and $\left\{\beta_{n}\right\}$ are sequences in $(0,1)$ satisfying the following conditions:

(C1) $\lim _{n \rightarrow \infty} \epsilon_{n}=0, \sum_{n=1}^{\infty} \epsilon_{n}=\infty$,

(C2) $\lim _{n \rightarrow \infty} \alpha_{n}=\lim _{n \rightarrow \infty} \varphi_{n}=0$,

(C3) $0<\liminf _{n \rightarrow \infty} \beta_{n} \leq \lim \sup _{n \rightarrow \infty} \beta_{n}<1$,

(C4) $\lim _{n \rightarrow \infty}\left|\lambda_{n+1}-\lambda_{n}\right|=\lim _{n \rightarrow \infty}\left|\delta_{n+1}-\delta_{n}\right|=\lim _{n \rightarrow \infty}\left|\tau_{n+1}-\tau_{n}\right|=0$,

(C5) $\left\{\tau_{n}\right\},\left\{\lambda_{n}\right\},\left\{\delta_{n}\right\} \subset[a, b]$ for some $a, b$ with $0 \leq a \leq b \leq 2\left(v-u \xi^{2}\right) / \xi^{2}, v>u \xi^{2}$.

Then, $\left\{x_{n}\right\}$ converges strongly to a point $z \in \Theta$, where $z=P_{\Theta}(I-A+\gamma f)(z)$, which solves the variational inequality

$$
\langle(A-\gamma f) z, x-z\rangle \geq 0, \quad \forall x \in \Theta,
$$


which is the optimality condition fot the minimization problem

$$
\min _{x \in \Theta}\left\{\frac{1}{2}\langle A x, x\rangle-h(x)\right\}
$$

where $h$ is a potential function for $\gamma f$ (i.e., $h^{\prime}(x)=\gamma f(x)$ for $x \in H$ ).

Proof. Put $D=0, F(x, y)=0$ for all $x, y \in E$ and $r_{n}=1$ for all $n \in \mathbb{N}$ in Theorem 3.3. Then, we have $u_{n}=P_{C} x_{n}=x_{n}$. So, by Theorem 3.3, we can conclude the desired conclusion easily.

\section{Acknowledgments}

The authors would like to express their thanks to the Faculty of Science KMUTT Research Fund for their financial support. The first author was supported by the Faculty of Applied Liberal Arts RMUTR Research Fund and King Mongkut's Diamond scholarship for fostering special academic skills by KMUTT. The second author was supported by the Thailand Research Fund and the Commission on Higher Education under Grant no. MRG5180034. Moreover, the authors are extremely grateful to the referees for their helpful suggestions that improved the content of the paper.

\section{References}

[1] G. Stampacchia, "Formes bilinéaires coercitives sur les ensembles convexes," Comptes Rendus Academy of Sciences, vol. 258, pp. 4413-4416, 1964.

[2] D. Gabay, "Applications of the method of multipliers to variational inequalities," in Augmented Lagrangian Methods, M. Fortin and R. Glowinski, Eds., pp. 299-331, North-Holland, Amsterdam, The Netherlands, 1983.

[3] M. A. Noor and W. Oettli, "On general nonlinear complementarity problems and quasi-equilibria," Le Matematiche, vol. 49, no. 2, pp. 313-331, 1994.

[4] E. Blum and W. Oettli, "From optimization and variational inequalities to equilibrium problems," The Mathematics Student, vol. 63, no. 1-4, pp. 123-145, 1994.

[5] S. D. Flåm and A. S. Antipin, "Equilibrium programming using proximal-like algorithms," Mathematical Programming, vol. 78, no. 1, pp. 29-41, 1997.

[6] X. Qin, M. Shang, and Y. Su, "Strong convergence of a general iterative algorithm for equilibrium problems and variational inequality problems," Mathematical and Computer Modelling, vol. 48, no. 7-8, pp. 1033-1046, 2008.

[7] Y. Yao, Y.-C. Liou, and J.-C. Yao, "Convergence theorem for equilibrium problems and fixed point problems of infinite family of nonexpansive mappings," Fixed Point Theory and Applications, vol. 2007, Article ID 64363, 12 pages, 2007.

[8] W. Takahashi, Nonlinear Functional Analysis, Fixed Point Theory and Its Applications, Yokohama, Yokohama, Japan, 2000.

[9] R. U. Verma, "Generalized system for relaxed cocoercive variational inequalities and projection methods," Journal of Optimization Theory and Applications, vol. 121, no. 1, pp. 203-210, 2004.

[10] R. U. Verma, "General convergence analysis for two-step projection methods and applications to variational problems," Applied Mathematics Letters, vol. 18, no. 11, pp. 1286-1292, 2005.

[11] R. T. Rockafellar, "On the maximality of sums of nonlinear monotone operators," Transactions of the American Mathematical Society, vol. 149, pp. 75-88, 1970.

[12] H. Iiduka and W. Takahashi, "Strong convergence theorems for nonexpansive mappings and inversestrongly monotone mappings," Nonlinear Analysis: Theory, Methods E Applications, vol. 61, no. 3, pp. 341-350, 2005.

[13] A. Moudafi and M. Théra, "Proximal and dynamical approaches to equilibrium problems," in Ill-Posed Variational Problems and Regularization Techniques (Trier, 1998), vol. 477 of Lecture Notes in Economics and Mathematical Systems, pp. 187-201, Springer, Berlin, Germany, 1999. 
[14] X. Qin, Y. J. Cho, and S. M. Kang, "Viscosity approximation methods for generalized equilibrium problems and fixed point problems with applications," Nonlinear Analysis: Theory, Methods $\mathcal{E}$ Applications, vol. 72, no. 1, pp. 99-112, 2010.

[15] Y. J. Cho, X. Qin, and S. M. Kang, "Some results for equilibrium problems and fixed point problems in Hilbert spaces," Journal of Computational Analysis and Applications, vol. 11, no. 2, pp. 294-316, 2009.

[16] Y. J. Cho, X. Qin, and J. I. Kang, "Convergence theorems based on hybrid methods for generalized equilibrium problems and fixed point problems," Nonlinear Analysis: Theory, Methods E Applications, vol. 71, no. 9, pp. 4203-4214, 2009.

[17] C. S Hu and G. Cai, "Viscosity approximation schemes for fixed point problems and equilibrium problems and variational inequality problems," Nonlinear Analysis: Theory, Methods E Applications, vol. 72, no. 3-4, pp. 1792-1808, 2010.

[18] N.-J. Huang, H.-Y. Lan, and K. L. Teo, “On the existence and convergence of approximate solutions for equilibrium problems in Banach spaces," Journal of Inequalities and Applications, vol. 2007, Article ID 17294, 14 pages, 2007.

[19] C. Jaiboon and P. Kumam, "Strong convergence theorems for solving equilibrium problems and fixed point problems of $\xi$-strict pseudo-contraction mappings by two hybrid projection methods," Journal of Computational and Applied Mathematics. In press.

[20] C. Jaiboon and P. Kumam, "A hybrid extragradient viscosity approximation method for solving equilibrium problems and fixed point problems of infinitely many nonexpansive mappings," Fixed Point Theory and Applications, vol. 2009, Article ID 374815, 32 pages, 2009.

[21] C. Jaiboon, W. Chantarangsi, and P. Kumam, "A convergence theorem based on a hybrid relaxed extragradient method for generalized equilibrium problems and fixed point problems of a finite family of nonexpansive mappings," Nonlinear Analysis: Hybrid Systems, vol. 4, no. 1, pp. 199-215, 2010.

[22] A. Kangtunyakarn and S. Suantai, "A new mapping for finding common solutions of equilibrium problems and fixed point problems of finite family of nonexpansive mappings," Nonlinear Analysis: Theory, Methods E Applications, vol. 71, no. 10, pp. 4448-4460, 2009.

[23] Q.-Y. Liu, W.-Y. Zeng, and N.-J. Huang, "An iterative method for generalized equilibrium problems, fixed point problems and variational inequality problems," Fixed Point Theory and Applications, vol. 2009, Article ID 531308, 20 pages, 2009.

[24] A. Moudafi, "Weak convergence theorems for nonexpansive mappings and equilibrium problems," Journal of Nonlinear and Convex Analysis, vol. 9, no. 1, pp. 37-43, 2008.

[25] J.-W. Peng, Y. Wang, D. S. Shyu, and J.-C. Yao, "Common solutions of an iterative scheme for variational inclusions, equilibrium problems, and fixed point problems," Journal of Inequalities and Applications, vol. 2008, Article ID 720371, 15 pages, 2008.

[26] X. Qin, M. Shang, and Y. Su, "A general iterative method for equilibrium problems and fixed point problems in Hilbert spaces," Nonlinear Analysis: Theory, Methods E Applications, vol. 69, no. 11, pp. 3897-3909, 2008.

[27] S. Takahashi and W. Takahashi, "Strong convergence theorem for a generalized equilibrium problem and a nonexpansive mapping in a Hilbert space," Nonlinear Analysis: Theory, Methods E Applications, vol. 69, no. 3, pp. 1025-1033, 2008.

[28] W.-Y. Zeng, N.-J. Huang, and C.-W. Zhao, "Viscosity approximation methods for generalized mixed equilibrium problems and fixed points of a sequence of nonexpansive mappings," Fixed Point Theory and Applications, vol. 2008, Article ID 714939, 15 pages, 2008.

[29] P. L. Combettes and S. A. Hirstoaga, "Equilibrium programming in Hilbert spaces," Journal of Nonlinear and Convex Analysis, vol. 6, pp. 117-136, 2005.

[30] W. Takahashi and M. Toyoda, "Weak convergence theorems for nonexpansive mappings and monotone mappings," Journal of Optimization Theory and Applications, vol. 118, no. 2, pp. 417-428, 2003.

[31] M. Shang, Y. Su, and X. Qin, "Strong convergence theorem for nonexpansive mappings and relaxed cocoercive mappings," International Journal of Applied Mathematics and Mechanics, vol. 3, no. 4, pp. 2434, 2007.

[32] F. Deutsch and I. Yamada, "Minimizing certain convex functions over the intersection of the fixed point sets of nonexpansive mappings," Numerical Functional Analysis and Optimization, vol. 19, no. 1-2, pp. 33-56, 1998. 
[33] I. Yamada, "The hybrid steepest descent method for the variational inequality problem over the intersection of fixed point sets of nonexpansive mappings," in Inherently Parallel Algorithms in Feasibility and Optimization and Their Applications (Haifa, 2000), D. Butnariu, Y. Censor, and S. Reich, Eds., vol. 8 of Studies in Computational Mathematics, pp. 473-504, North-Holland, Amsterdam, The Netherlands, 2001.

[34] H.-K. Xu, "Iterative algorithms for nonlinear operators," Journal of the London Mathematical Society, vol. 66, no. 1, pp. 240-256, 2002.

[35] H. K. Xu, "An iterative approach to quadratic optimization," Journal of Optimization Theory and Applications, vol. 116, no. 3, pp. 659-678, 2003.

[36] G. Marino and H.-K. Xu, "A general iterative method for nonexpansive mappings in Hilbert spaces," Journal of Mathematical Analysis and Applications, vol. 318, no. 1, pp. 43-52, 2006.

[37] S.-S. Chang, H. W. J. Lee, and C. K. Chan, "A new method for solving equilibrium problem fixed point problem and variational inequality problem with application to optimization," Nonlinear Analysis: Theory, Methods E Applications, vol. 70, no. 9, pp. 3307-3319, 2009.

[38] Y. Yao, M. A. Noor, and Y.-C. Liou, “On iterative methods for equilibrium problems," Nonlinear Analysis: Theory, Methods \& Applications, vol. 70, no. 1, pp. 497-509, 2009.

[39] Y. J. Cho and X. Qin, "Generalized systems for relaxed cocoercive variational inequalities and projection methods in Hilbert spaces," Mathematical Inequalities $\mathcal{E}$ Applications, vol. 12, no. 2, pp. 365375, 2009.

[40] Z. Opial, "Weak convergence of the sequence of successive approximations for nonexpansive mappings," Bulletin of the American Mathematical Society, vol. 73, pp. 591-597, 1967.

[41] K. Shimoji and W. Takahashi, "Strong convergence to common fixed points of infinite nonexpansive mappings and applications," Taiwanese Journal of Mathematics, vol. 5, no. 2, pp. 387-404, 2001.

[42] T. Suzuki, "Strong convergence of Krasnoselskii and Mann's type sequences for one-parameter nonexpansive semigroups without Bochner integrals," Journal of Mathematical Analysis and Applications, vol. 305, no. 1, pp. 227-239, 2005.

[43] H.-K. Xu, "Viscosity approximation methods for nonexpansive mappings," Journal of Mathematical Analysis and Applications, vol. 298, no. 1, pp. 279-291, 2004. 